\title{
Collision Patterns on Mollusc Shells
}

\author{
P.J. PLATH*, J.K. PLATH and J. SCHWIETERING \\ Institute of Applied and Physical Chemistry, University of Bremen, Bibliothekstraße NW 2 D-28334 Bremen, Germany
}

(Received 9 October 1996)

\begin{abstract}
On mollusc shells one can find famous patterns. Some of them show a great resemblance to the soliton patterns in one-dimensional systems. Other look like Sierpinsky triangles or exhibit very irregt:ar patterns. Meinhardt has shown that those patterns can be well described by reaction-diffusion systems [1]. However, such a description neglects the discrete character of the cell system at the growth front of the molluse shell.

We have therefore developed a one-dimensional cellular vector automaton model which takes into account the cellular behaviour of the system [2]. The state of the mathematical cell is defined by a vector with two components. We looked for the most simple transformation rules in order to develop quite different types of waves: classical waves, chemical waves and different types of solitons. Our attention was focussed on the properties of the system created through the collision of two waves.
\end{abstract}

Keywords: Cellular automata, Chemical waves, Solitons, Sea shell patterns

\section{A INTRODUCTION}

One example is the sea shell pattern of the Amoria dampiera (Fig. 1), which seems to be very simple. Stripes perpendicular to the direction of growth look like waves. One gets the impression that all the cells at the growing edge (the lip of the shell) are oscillating in synchrony. The question that arises is how are they synchronized?

Meinhardt modelled this pattern using his famous activator-inhibitor model of two coupled partial differential equations of the reaction diffusion type [1]. Here $a$ denotes the activator and $b$ the inhibitor concentration.

$$
\begin{aligned}
& \frac{\partial a}{\partial t}=s\left(\frac{a^{2}}{b}+b_{a}\right)-r_{a} a+D_{a} \frac{\partial^{2} a}{\partial x^{2}}, \\
& \frac{\partial b}{\partial t}=s a^{2}-r_{b} b+b_{b}+D_{b} \frac{\partial^{2} b}{\partial x^{2}} .
\end{aligned}
$$

The constants $b_{a}$ and $b_{b}$ are the permanent activator and inhibitor productions, while $s$ is the so-called "source density" of the activator production. For a given set of parameters this system of differential equations will exhibit stripes perpendicular to the direction of growth. But

* Corresponding author. 


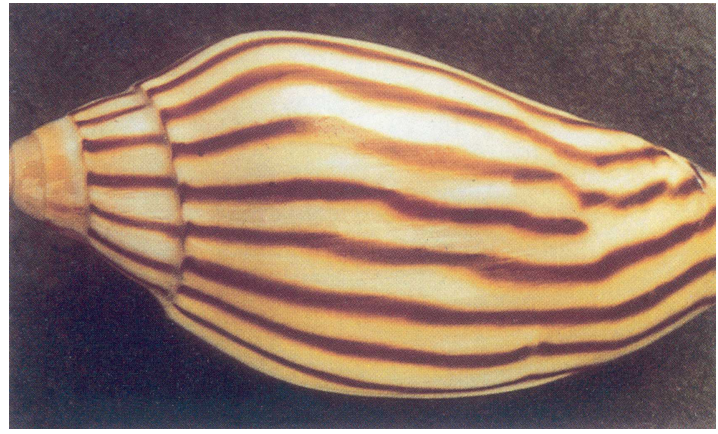

FIGURE 1 Pattern of the sea shell of Amoria damperia (with the kind permission of Meinhardt [1]). See Color Plate I.

models of this type of spatially non-restricted kinetic reaction-diffusion equations totally neglect the cellular character of the biological system.

During the "13. Winterseminar on Zeinisjoch (March 1996)", G. Baier presented a system comprising a large number of linearly arranged diffusively coupled chemical oscillators. It is very large-scaled to handle such a system, but seems to permit the description of pattern formation in the biological system of the sea shells as well.

However, our basic question is, "Can we construct a cellular automaton model, discrete in space and time, which is able to show the patterns being observed in the animals?"

\section{B AVERAGING AND DIFFUSION}

To answer this question, we first have to ask "What does diffusion mean in a cellular system?" Moreover, we have to ask whether random events are necessary in order to understand diffusion. Let us give a very first and rough answer to both questions. In the linear case, one does not need randomness to describe isotropic diffusion processes, due to the fact that the linear space is isotropic by definition. So diffusion becomes any kind of spatial averaging. For example, let us take Fick's second law

$$
\frac{\partial c}{\partial \tau}=D_{c} \frac{\partial^{2} c}{\partial x^{2}}
$$

As usual, one can rewrite the Laplacian term by the well known spatial discretisation

$$
\nabla^{2} c=c(x-1, \tau)-2 c(x, \tau)+c(x+1, \tau) .
$$

So Fick's law can be formulated by the iterative equation, where $t$ is the discrete time now and $c$ the concentration. Let us call this discrete formulation of Fick's second law the Laplacian difiusion:

$$
\begin{aligned}
c(x, t+1)= & c(x, t)+D_{c}[c(x-1, t)-2 c(x, t) \\
& +c(x+1, t)], \quad x \in \mathcal{Z} ; t \in \mathcal{N} .
\end{aligned}
$$

Figure 2 shows an example of how this iterative equation works.

On the other hand, there is the well-known binomial smoothing procedure of a discrete function $Z(i)$ with $i \in \mathcal{N}$ :

$$
\begin{aligned}
Z(i, n+1)= & \frac{1}{4}(Z(i-1, n)+2 Z(i, n) \\
& +Z(i+1, n)), \quad n=0,1,2 \ldots,
\end{aligned}
$$

where $n$ is the number of smoothing generation.

Interpreting this number $n$ as discrete time $t$, this smoothing procedure formally represents a temporal averaging. One can easily rewrite this smoothing procedure in terms of the diffusion equation, replacing $Z(i, n)$ by $c(x, t)$ :

$$
\begin{aligned}
c(x, t+1)= & \frac{1}{4}(c(x-1, t)+2 c(x, t)+c(x+1, t)) \\
& +\frac{2}{4} c(x, t)-\frac{2}{4} c(x, t) \\
= & c(x, t)+\frac{1}{4}(c(x-1, t)-2 c(x, t)+c(x+1, t)) \\
= & c(x, t)+D_{c}(c(x-1, t)-2 c(x, t)+c(x+1, t))
\end{aligned}
$$

with the diffusion coefficient $D_{c}=\frac{1}{4}$. Let us call this special procedure the binomial diffusion (Fig. 3). We can now state that, in the case of a linear cellular automaton, diffusion means any kind of a spatial averaging of the states of the cells over time. In order to deal with natural numbers only, we define integral states $z(i, t)$ by means of the Gaussian-brackets notation $[u]$ :

$$
z(i, t+1)=[\hat{z}(i, t)]
$$




\section{Laplacian diffusion - Fick's second law}

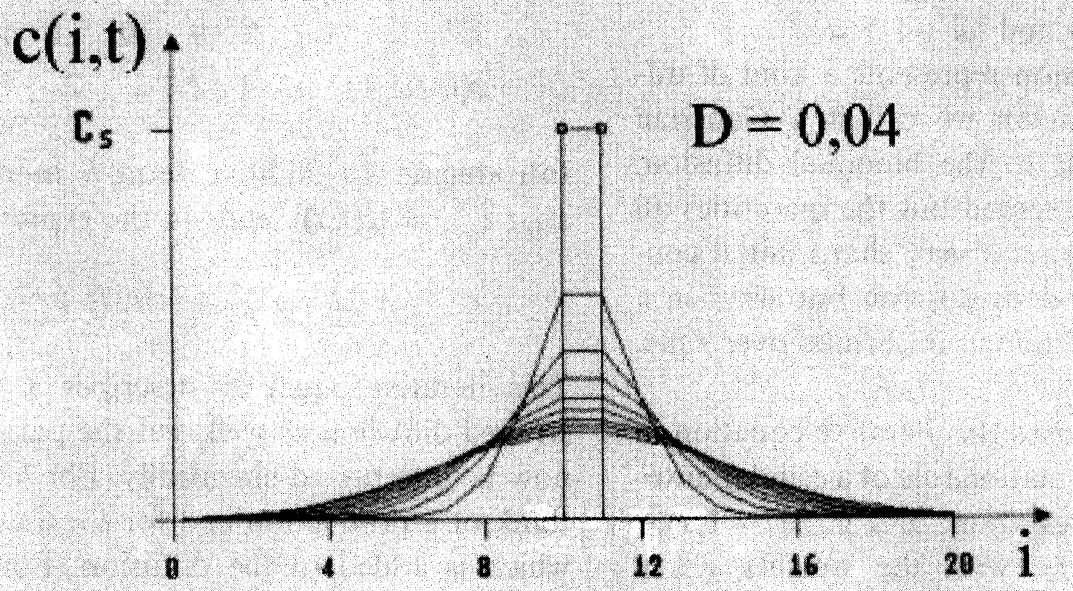

FIGURE 2 Graphs of the iterative formulation of Fick's second law in one-dimensional space.

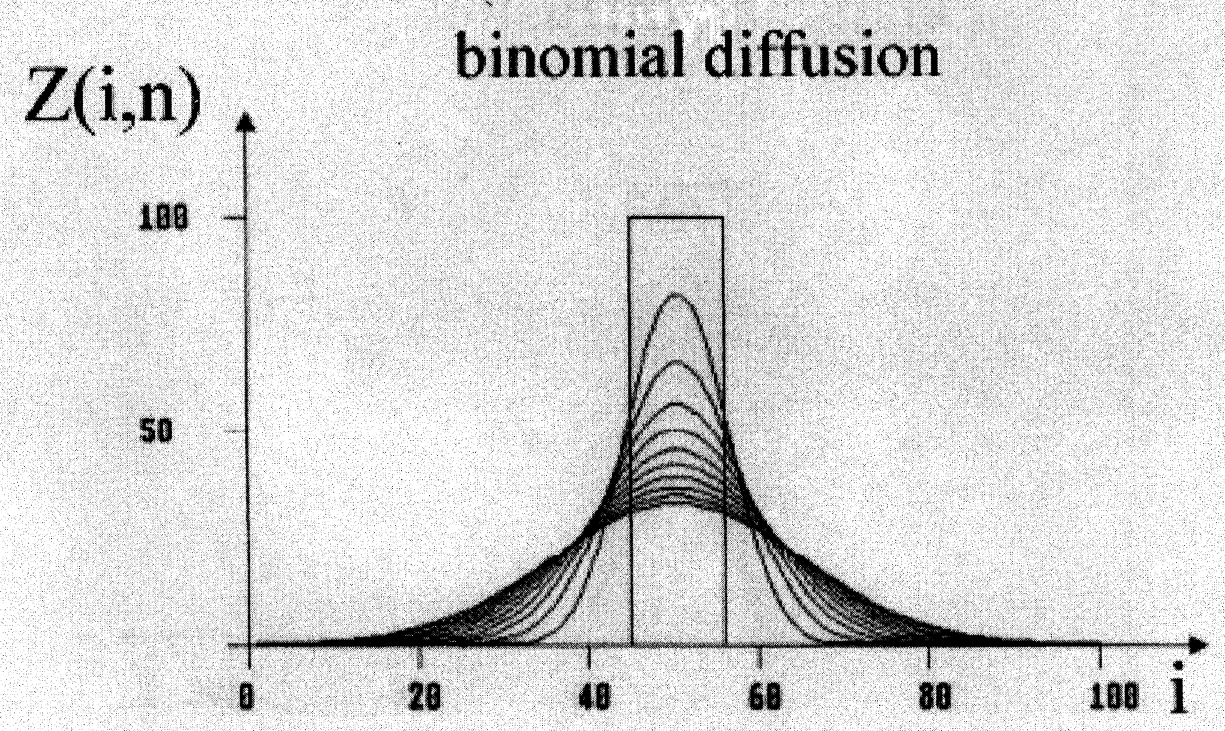

FIGURE 3 Graphs of the binomial diffusion of a sharp concentration profile at the beginning. 
with

$$
\hat{z}(i, t)=\frac{1}{4}(z(i-1, t)+2 z(i, t)+z(i+1, t)),
$$

where $[u]$ is the largest natural number that is less or equal to $u:[u] \leq u$ and $[u]+1>u$.

Again, this expression represents a kind of diffusion, for which reason we call it a Gaussian diffusion. In contrast to the binomial diffusion, however, it does not spread out the quantities all over the space. If we set a very sharp initial concentration profile, it does not run but stays in a restricted domain, although it shrinks over time. (see Fig. 4)

One can easily express this iterative equation in the form of a transformation rule of a cellular automaton with the abbreviation: $\Sigma \equiv g_{-1} z(i-1, t)+$ $g_{0} z(i, t)+g_{+1} z(i+1, t)$; with the weights $g_{-1}=$ $g_{+1}=1, g_{0}=2$ as shown in Table I:
TABLE I

$$
\begin{array}{lllllllll}
\Sigma & 0 & 1 & 2 & 3 & 4 & 5 & 6 & \\
z(i, t+1) & 0 & 0 & 0 & 0 & 1 & 1 & 1 & \\
& & & & & & & \\
\Sigma & 7 & 8 & 9 & 10 & 11 & 12 & \ldots \\
z(i, t+1) & 1 & 2 & 2 & 2 & 2 & 3 & \ldots
\end{array}
$$

Introducing an additive term $b$ in the equation $z(i, t+1)=[\hat{z}(i, t)]$ leads to the expression:

$$
z_{b}(i, t+1)=[\hat{z}(i, t)+b] .
$$

This iterative equation describes a spatially restricted diffusion as well, but the parameter $b$ can now be interpreted chemically. For $b>0$ one may think of a production and for $b<0$ a destruction, which is added to the diffusion. For example if $b=0.25$ diffusion will be stopped after some time

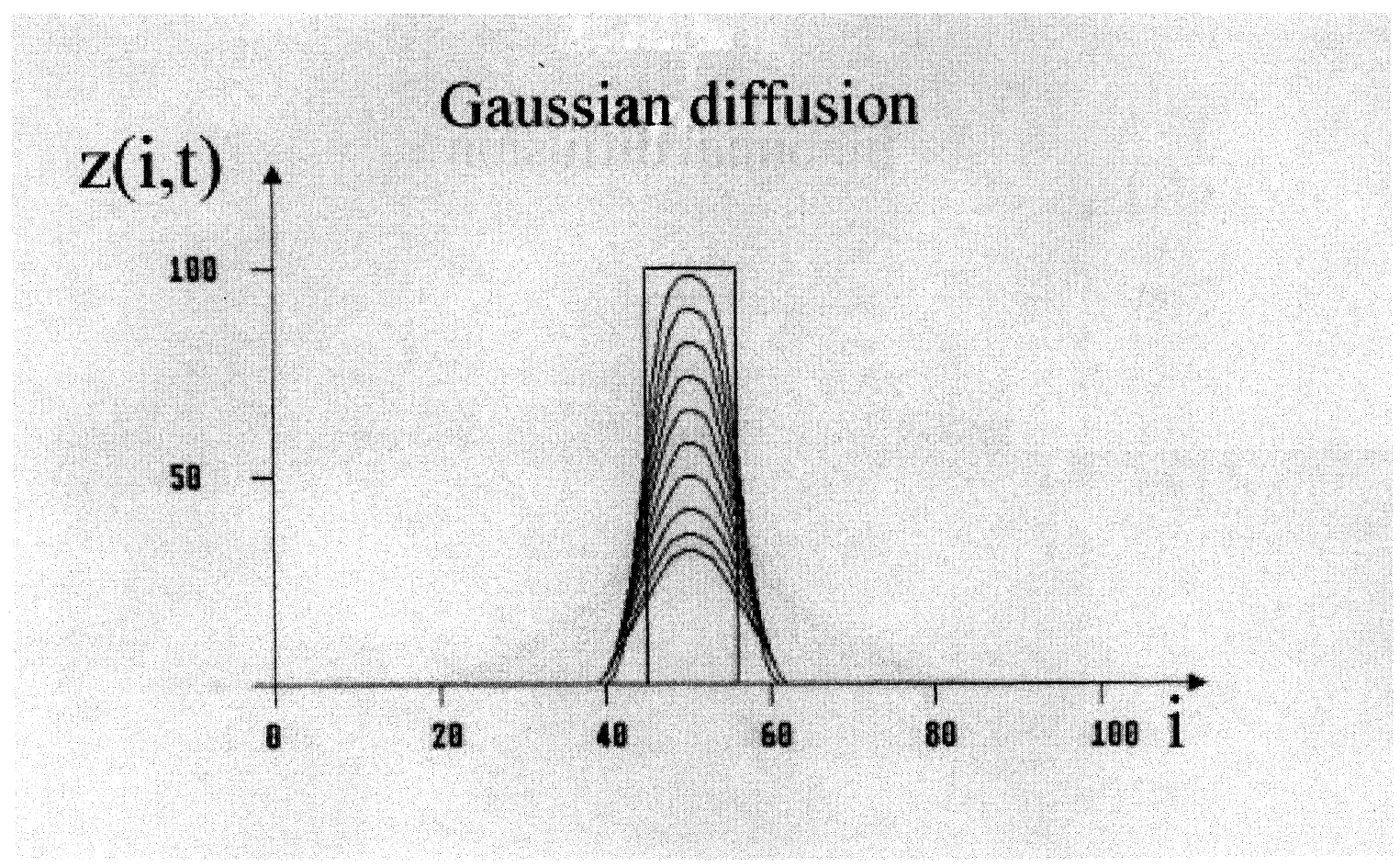

FIGURE 4 Graph of the spatially restricted Gaussian diffusion. 


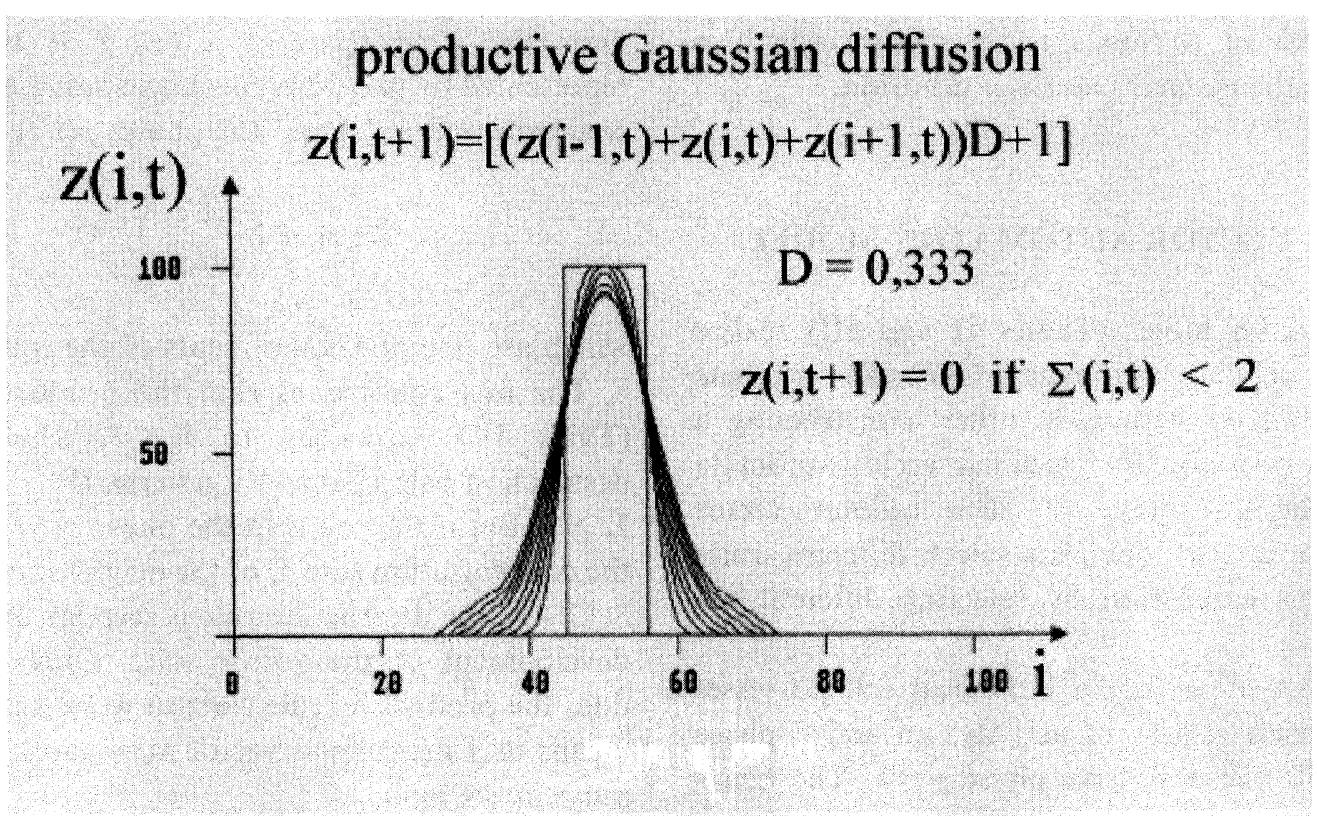

FIGURE 5 Development of the productive Gaussian diffusion with the limitation of the state growth if $z(i, t)=0$ and $\Sigma \leq L$.

by the production (Fig. 5). One obtains a stationary spatial distribution of states: $z(i, t+1)=$ $z(i, t)$ for all cells $i$. Let us call $G$ the sum of weights of states of the neighbouring cells:

$$
G=\sum_{k=-1}^{+1} g_{k}
$$

Increasing $b$, the stationary state is reached if $b$ becomes larger or equal to $1 / G: 1 / G \leq b<1$.

Setting $b=1$ it generally means a shift of the $z(i, t+1)$-row of Table I to the left by four digits:

$$
z_{1}(i, t+1)=[\hat{z}(i, t)+1]=\left[\frac{1}{4} \Sigma+1\right]:
$$

\section{TABLE II}

$$
\begin{array}{lcccccccc}
\Sigma & 0 & 1 & 2 & 3 & 4 & 5 & 6 & \\
z(i, t+1) & 0 & 1 & 1 & 1 & 2 & 2 & 2 & \\
\Sigma & 7 & 8 & 9 & 10 & 11 & 12 & \ldots \\
z(i, t+1) & 2 & 3 & 3 & 3 & 3 & 4 & \ldots
\end{array}
$$

The production now exceeds the diffusion and the states will grow anywhere, even if $z(i, t)=0$.
Introducing a lower limit $L$ for opening the state growth of a cell:

$z(i, t+1)= \begin{cases}0 & \text { if } z(i, t)=0 \text { and } \Sigma \leq L, \\ {[\hat{z}(i, t)+b]} & \text { otherwise, }\end{cases}$

the states of those cells start to grow which have an appropriate neighbourhood (see Fig. 5). As a result, Gaussian diffusion is not spatially restricted anywhere.

If $b$ equals minus one: $b=-1$ the future states become

$$
z_{-1}(i, t+1)=[\hat{z}(i, t)-1]
$$

and the lower row of the transformation rule (Table I) is shifted to the right.

\section{TABLE III}

$$
\begin{array}{lllllllll}
\Sigma & 0 & 1 & 2 & 3 & 4 & 5 & 6 & \\
z(i, t+1) & 0 & 0 & 0 & 0 & 0 & 0 & 0 & \\
\Sigma & 7 & 8 & 9 & 10 & 11 & 12 & \ldots \\
z(i, t+1) & 0 & 1 & 1 & 1 & 1 & 2 & \ldots
\end{array}
$$


This value of $b$ causes a destruction which is added to the normal Gaussian diffusion.

\section{THE VECTOR AUTOMATON MODEL}

Both look-up tables (Tables II and III) realize classical cellular automata. One can combine these automata with each other, constructing a new type of automata - cellular vector automata [2-5]. The advantage of such cellular vector automata is that one can select different rules within one expression by selecting different values of $b$.

Let us assume that a biological cell possesses two different phases of activity: an active phase with $p=0$ and a passive phase $p=1$. The behaviour of the biological cells in these two phases should be characterized by a production $(p=0$; $b>0)$ in the active state, and a destruction $(p=1$; $b \leq 0$ ) in the passive state. So the phases may be used to construct a switch between the different rules. But it should be an internal switch, the position of which should again depend on the states of the neighbouring cells.

For example, if $p(i, t)=0$ then:

\section{TABLE IV}

$$
\begin{array}{lllllllll}
\Sigma & 0 & 1 & 2 & 3 & 4 & 5 & 6 & \\
p(i, t+1) & 0 & 0 & 0 & 0 & 0 & 0 & 0 & \\
\Sigma & 7 & 8 & 9 & 10 & 11 & 12 & \ldots \\
p(i, t+1) & 0 & 1 & 1 & 1 & 1 & 1 & \ldots
\end{array}
$$

and if $p(i, t)=1$

\section{TABLE V}

$$
\begin{array}{lllllllll}
\Sigma & 0 & 1 & 2 & 3 & 4 & 5 & 6 & \\
p(i, t+1) & 0 & 0 & 0 & 0 & 1 & 1 & 1 & \\
\Sigma & 7 & 8 & 9 & 10 & 11 & 12 & \ldots \\
p(i, t+1) & 1 & 1 & 1 & 1 & 1 & 1 & \ldots
\end{array}
$$

Now the state $\vec{z}(i, t)$ of a cell $i$ at time $t$ is represented by two different quantities: the integral concentration $z(i, t)$ and the phases $p(i, t)$ :

$$
\vec{z}(i, t)=\left(\begin{array}{c}
z(i, t) \\
p(i, t)
\end{array}\right),
$$

which are now the components of the state vector $\vec{z}$. The first rule means that the production rule (Table IV) works up to $\Sigma \leq 7$, whereas the destruction rule (Table V) governs the region for $\Sigma \geq 8$. But if the cell is in the phase $p(i, t)=1$ and the concentration sum $\Sigma$ of the neighbours exceeds 3: $\Sigma \geq 4$, the destruction rule is responsible for the development of the vector state. Only if $\Sigma \leq 3$ does the production rule start to work again. This means that the cellular vector automaton displays a hysteresis loop.

\section{DISSIPATION}

The system of coupled differential equations, used by Meinhardt [1] is essentially dissipative, as can be shown by the non-vanishing trace of the Jacobian. In cellular automata there is no explicit expression for this proof of the dissipative character of the transformation rule. But looking at the maps one can decide whether the system is conservative or dissipative. For example, if there is a one-to-one map the system is conservative [6]. But in our case the map

$$
F: \vec{z}(i, t) \longmapsto \vec{z}(i, t+1)
$$

is not unique. The graph of this map is composed of two tent-like staircases, which are shifted against each other. So we have a manyto-one map which is essentially dissipative.

Since the general transformation rule of the vector state $\vec{z}(i, t)$ realizes a dissipative map, the cellular vector automaton becomes a powerful tool for modelling the patterns of a natural system such as mollusc shells. 
For example, using the transformation rule

\section{TABLE VI}

$$
\begin{aligned}
& \begin{array}{clllllll}
\Sigma & 0 & 1 & 2 & 3 & 4 & 5 \\
p(i, t)=0 & z(i, t+1) & 0 & 1 & 1 & 2 & 2 & 2 \\
p(i, t)=1 & z(i, t+1) & 0 & 0 & 0 & 0 & 0 & 0 \\
p(i, t)=0 & p(i, t+1) & 0 & 0 & 0 & 0 & 0 & 0 \\
p(i, t)=1 & p(i, t+1) & 0 & 0 & 0 & 0 & 0 & 0
\end{array} \\
& \begin{array}{lllllll}
\Sigma & 6 & 7 & 8 & 9 & 10 & 11
\end{array} \\
& p(i, t)=0 \quad z(i, t+1) \quad 3 \quad 3 \quad 3 \quad r \begin{array}{lllll} 
& 4
\end{array}
\end{aligned}
$$

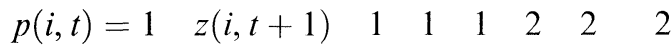

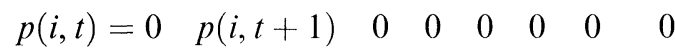

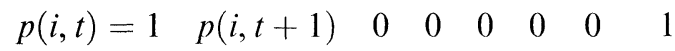

$$
\begin{aligned}
& \begin{array}{clllll} 
& \Sigma & 12 & \ldots & 63 & 64 \\
p(i, t)=0 & z(i, t+1) & 5 & \ldots & 21 & 22 \\
p(i, t)=1 & z(i, t+1) & 3 & \ldots & 19 & 20 \\
p(i, t)=0 & p(i, t+1) & 0 & \ldots & 0 & 1 \\
p(i, t)=1 & p(i, t+1) & 1 & \ldots & 1 & 1
\end{array}
\end{aligned}
$$

and starting with all cells being in the vector state

$$
\vec{z}(i, 0)=\left(\begin{array}{c}
z(i, 0) \\
p(i, 0)
\end{array}\right)=\left(\begin{array}{l}
0 \\
0
\end{array}\right)
$$

except the cells $j, k, l, \ldots$, which are in the state, for example

$$
\vec{z}(j, 0)=\left(\begin{array}{c}
z(j, 0) \\
p(j, 0)
\end{array}\right)=\left(\begin{array}{l}
1 \\
0
\end{array}\right)
$$

the automaton runs into a limit cycle after a long time (Fig. 6). To construct this rule (Table VI), we used a weight vector $\vec{g}=\left(g_{-1}, g_{0}, g_{+1}\right)=$ $(1,1,1)$, and a vector $\vec{s}=(z(i-1, t), z(i, t)$, $z(i+1, t))$ of the neighboured concentrations so that $\Sigma$ becomes the scalar product $\Sigma=\vec{s} \vec{g}$ and the averaging procedure of the first row in the rule (Table VI) becomes: $z_{1}(i, t+1)=\left[\frac{1}{3} \Sigma+1\right]=$ $\left[\frac{1}{3} \vec{s} \vec{g}+1\right]$. This row represents a diffusion process

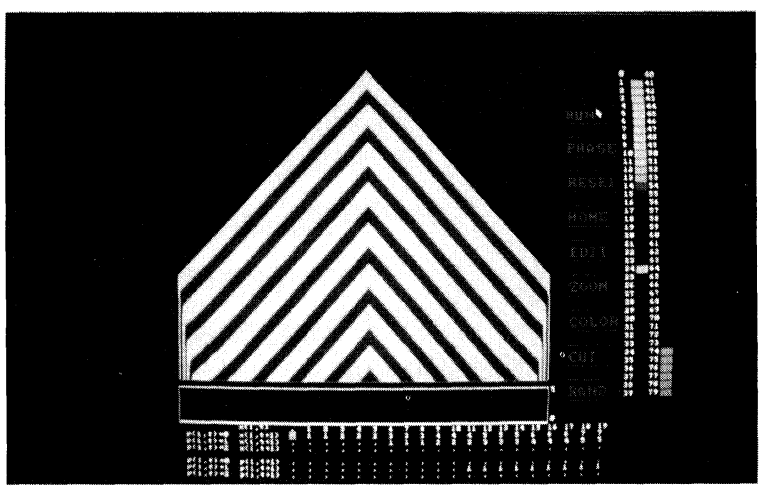

FIGURE 6 Spatio-temporal development of the automaton Table VI starting with one cell. The time axis runs from top to bottom.

as well, the diffusion coefficient of which is $D=\frac{1}{3}$ :

$$
\begin{aligned}
z(i, t+1)= & z(i, t)+\frac{1}{3}(z(i-1, t)-2 z(i, t) \\
& +z(i+1, t))
\end{aligned}
$$

In particular, if we use a finite number of 599 linearly arranged cells of the automaton and cyclic boundary conditions, the periodic state of the automaton is structured as riffled waves perpendicular to the direction of time (Fig. 7), which is also the direction of growth of the mollusc shell lip. Figure 8 shows that there are indeed mollusc shells which exhibit precisely such riffled patterns.

Rather than starting with only one cell, but instead with many cells $j$ in the states $\vec{z}(j, 0)=\left(\begin{array}{l}1 \\ 0\end{array}\right)$ distributed randomly, one obtains very plane waves, perpendicular to the direction of growth (Fig. 9). There are also sea shells which display precisely such patterns; (see Fig. 1).

The other very simple shell pattern Meinhardt discussed [1] involves stripes parallel to the direction of growth (Fig. 10). Using the vector $\vec{g}=(1,1,1)$ and a very strong production rule $z(i, t+1)=\left[\frac{1}{3} \Sigma+3\right]$ with the transformation matrix: 


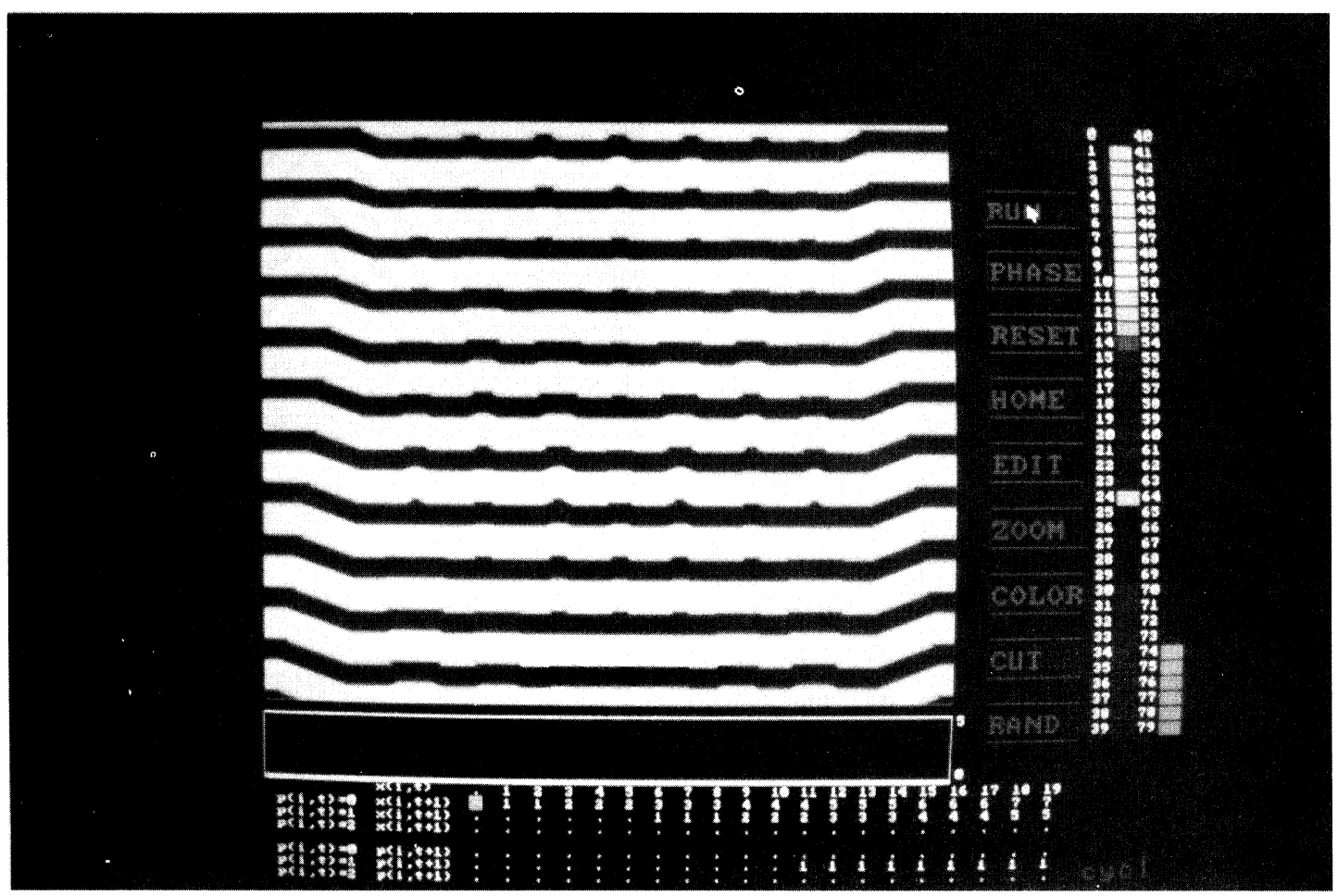

FIGURE 7 Spatio-temporal development of the automaton Table VI after long period of time. Riffled waves perpendicular to the direction of growth develop under cyclic boundary conditions.

\section{TABLE VII}

$$
\begin{array}{cllllllll}
p(i, t)=0 & z(i, t+1) & 0 & 3 & 3 & 4 & 4 & 4 & 5 \\
p(i, t)=1 & z(i, t+1) & 0 & 0 & 0 & 0 & 0 & 0 & 1 \\
p(i, t)=0 & p(i, t+1) & 0 & 0 & 0 & 0 & 1 & 0 & 0 \\
p(i, t)=1 & p(i, t+1) & 0 & 0 & 1 & 1 & 1 & 1 & 1 \\
& & & 7 & 8 & 9 & \ldots & 63 & 64 \\
p(i, t)=0 & z(i, t+1) & 5 & 5 & 6 & \ldots & 23 & 25 \\
p(i, t)=1 & z(i, t+1) & 1 & 1 & 2 & \ldots & 19 & 20 \\
p(i, t)=0 & p(i, t+1) & 0 & 0 & 0 & \ldots & 0 & 0 \\
p(i, t)=1 & p(i, t+1) & 1 & 1 & 1 & \ldots & 1 & 1
\end{array}
$$

and a few (about 20) arbitrary cells, after some time one obtains stripes parallel to the direction of growth (Fig. 11). These stripes represent spatially stable situations of the automaton. They

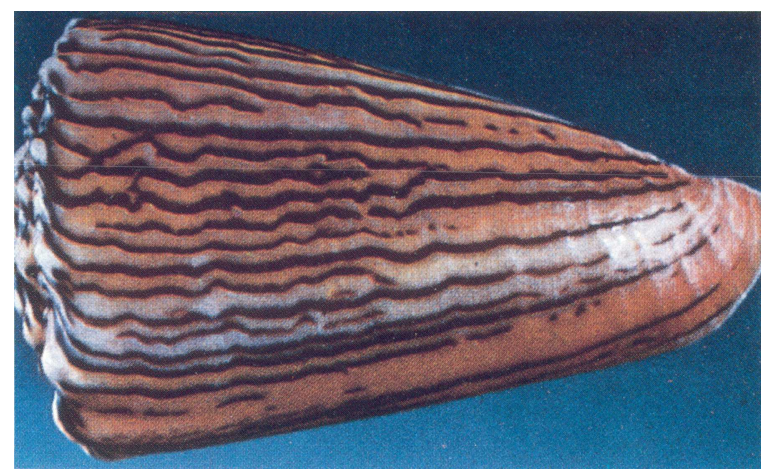

FIGURE 8 Pattern of riffled stripes (perpendicular to the direction of growth) on the cone shell principles (with the kind permission of Jerry G. Walls [7a]). See Color Plate II.

are created by cells which run into a non-vanishing stable "fixed point". Finally this means that one obtains a vector state distribution which is stable in space and time. 


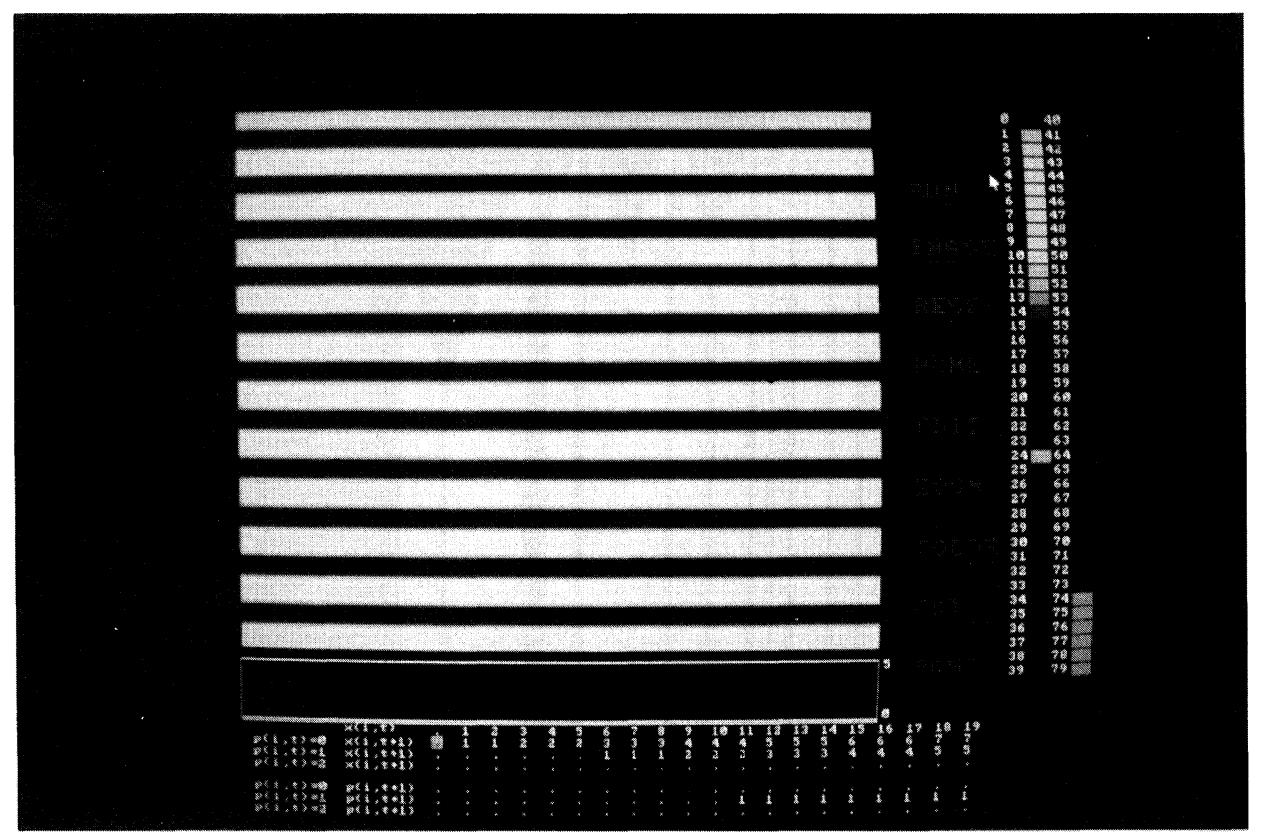

FIGURE 9 "Phase waves" in the automaton in Table VI with cyclic boundary conditions. They arise if one starts with hundreds of randomly distributed cells in the state $\vec{z}(j, 0)=\left(\begin{array}{l}1 \\ 0\end{array}\right)$.

But if one looks carefully at the origin of the stripes, one can see that they are created by the collision of solitary waves. If solitary waves collide, a new, localized "collision state" emerges. Such a state may be stable, as in the case of stripes parallel to the direction of growth. However, other solitary waves might exist the excited collision state of which is unstable and vanishes or it decomposes after a while. In the first case one should obtain "chemical waves", while in the second case "solitons" should be observed.

For example, there is a very simple automaton rule which results a chemical wave:

\section{TABLE VIII}

$$
\begin{array}{cccccccc} 
& \Sigma & 0 & 1 & 2 & 3 & 4 & \ldots \\
p(i, t)=0 & z(i, t+1) & 0 & 1 & 1 & 2 & 0 & \ldots \\
p(i, t)=1 & z(i, t+1) & 0 & 0 & 0 & 0 & 0 & \ldots \\
p(i, t)=0 & p(i, t+1) & 0 & 1 & 1 & 1 & 1 & \ldots \\
p(i, t)=1 & p(i, t+1) & 0 & 1 & 1 & 1 & 1 & \ldots
\end{array}
$$

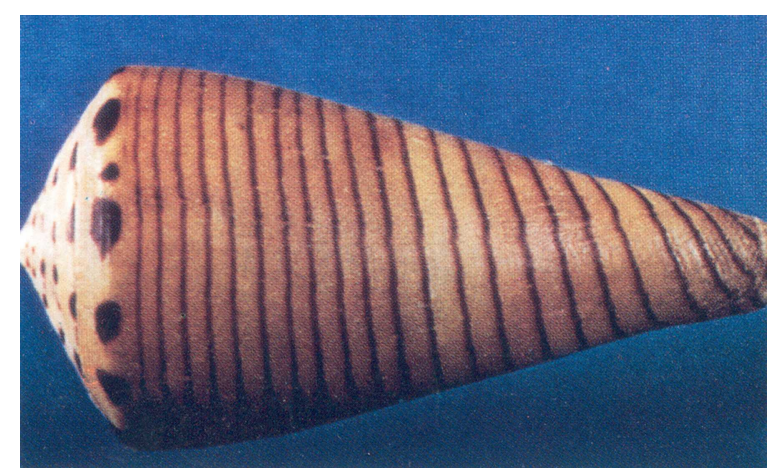

FIGURE 10 The pattern of the cone shell Hirasei (44.2 $\mathrm{mm}$ ) from Taiwan shows stripes parallel to the direction of growth (with the kind permission of Jerry G. Walls [7b]). See Color Plate III.

The excited state which is created by the collision vanishes after a few time steps. The zero state of the cells will form the only stable fixed point of the dynamics of the collision system (Fig. 12). This simple rule is nothing other 


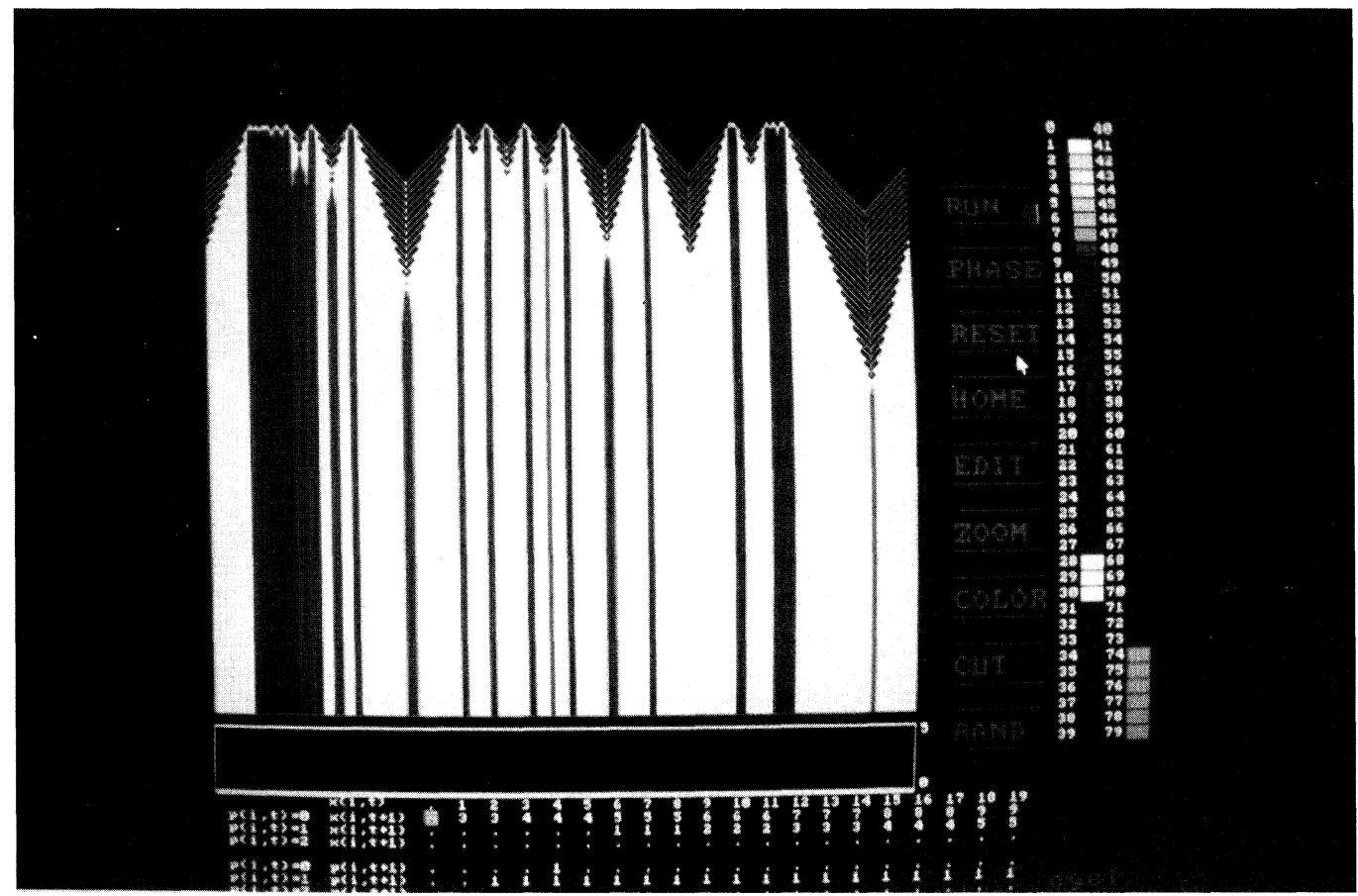

FIGURE 11 Development of the automaton in Table VII, causing stripes parallel to the direction of growth.

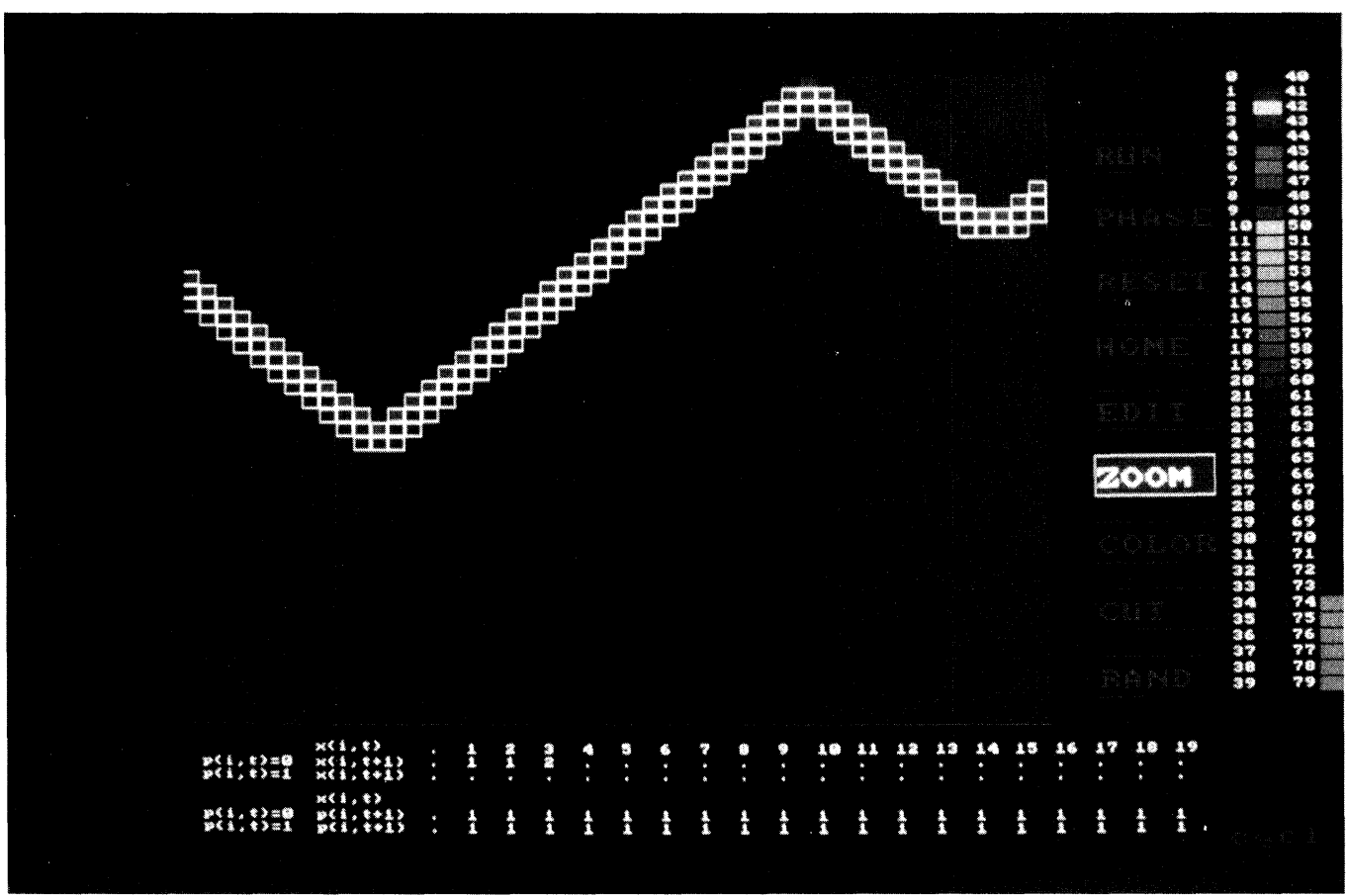

FIGURE 12 The spatio-temporal development of a "chemical wave" in the one-dimensional space of the automaton according to Table VIII. 
than a reformulation of the famous WienerRosenblueth automaton for a one-dimensional cellular automaton. It therefore comes as no surprise that some mollusc shells exhibit patterns which resemble the annihilation of colliding solitary waves (Fig. 13).

A closer look at such mollusc patterns reveals that this collision behaviour of chemical waves is often accompanied by soliton-like behaviour. If solitons collide, they pass each other by a phase shift. The following transformation matrix enables the creation of such solitons (Fig. 14):

\section{TABLE IX}

$$
\begin{array}{lllllll} 
& \Sigma & 0 & 1 & 2 & 3 & 4 \\
p(i, t)=0 & z(i, t+1) & 0 & 1 & 1 & 2 & 2 \\
p(i, t)=1 & z(i, t+1) & 0 & 0 & 0 & 0 & 0 \\
p(i, t)=0 & p(i, t+1) & 0 & 0 & 0 & 1 & 1 \\
p(i, t)=1 & p(i, t+1) & 0 & 0 & 1 & 1 & 1 \\
& \Sigma & 5 & 6 & 7 & \ldots \\
p(i, t)=0 & z(i, t+1) & 0 & 0 & 0 & \ldots \\
p(i, t)=0 & z(i, t+1) & 0 & 1 & 0 & \ldots \\
p(i, t)=0 & z(i, t+1) & 1 & 1 & 1 & \ldots \\
p(i, t)=0 & z(i, t+1) & 1 & 1 & 1 & \ldots
\end{array}
$$

These two different kinds of solitary waves are not the only ones, however. There is a variety of

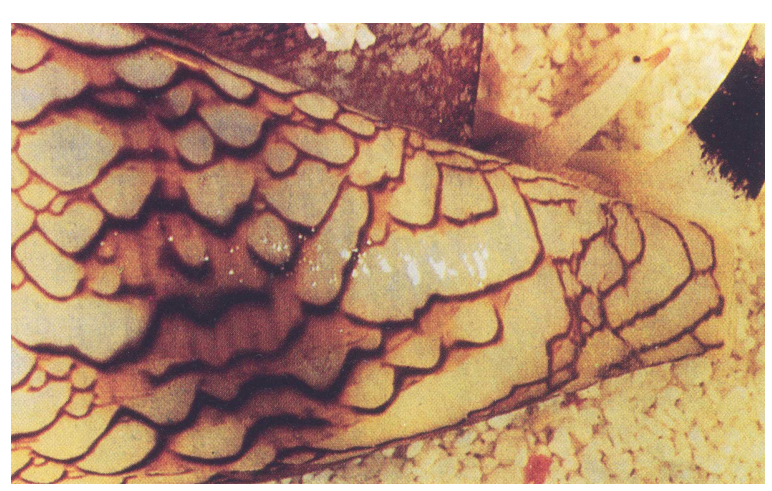

FIGURE 13 The cone shell Conus textile from the Great Barrier Reef (with the kind permission of Jerry G. Walls [7c]). See Color Plate IV. solitary waves the collision states of which differ considerably. In the case of the chemical and the soliton waves respectively, one can classify the solitary waves purely in terms of their collision behaviour. Classifying waves in this way is very fundamental [11]. For example, there are solitary waves which cross each other without any phase shift (Fig. 15). They can generated by the transfomation matrix:

\section{TABLE X}

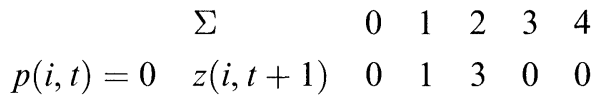

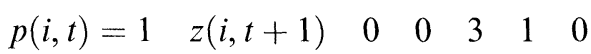

$$
\begin{aligned}
& p(i, t)=0 \quad p(i, t+1) \quad 0 \quad 110000 \\
& p(i, t)=1 \quad p(i, t+1) \quad 0 \quad 1 \quad 0011 \\
& \begin{array}{lllll}
\Sigma & 5 & 6 & 7 & \ldots
\end{array} \\
& p(i, t)=1 \quad z(i, t+1) \quad 0 \quad 0000 . \\
& p(i, t)=1 \quad z(i, t+1) \quad 0 \quad 0010 \quad \ldots
\end{aligned}
$$

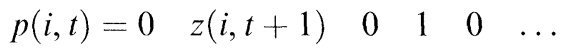

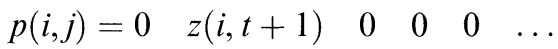

In this respect they behave like classical waves. In this case the collision state is very unstable. Its lifetime tends to zero.

\section{E STRUCTURES OF COLLISION}

As has been demonstrated, there are many structures for the collision states of solitary waves. The question arises as to whether one can create chaotic collision patterns as well. To answer this question, one should investigate the automata under consideration in greater detail. The procedure of averaging does not enable the creation of waves, even in the case of integral numbers, for example: $z(i, t+1)=\left[\frac{1}{3} \Sigma\right]$. However, taking a productive diffusion $z(i, t+1)=\left[\frac{1}{3} \Sigma+1\right]$ and in addition if $\Sigma(i, t)=0 \Longrightarrow z(i, t+1)=0$, one may obtain an automaton which will create a chemical wave with a saw-tooth profile. 


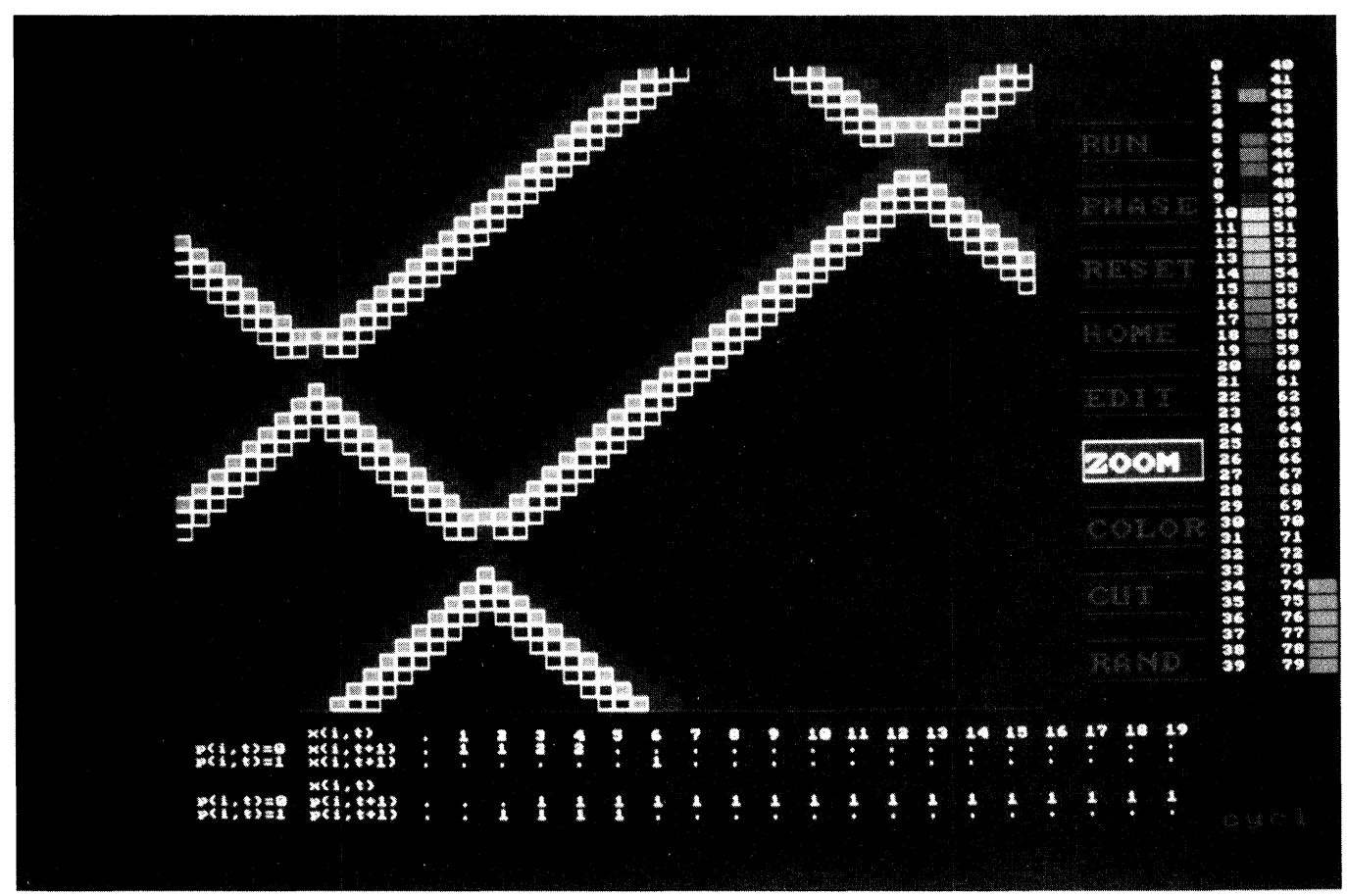

FIGURE 14 The automaton in Table IX, which creates solitons in the one-dimensional space.

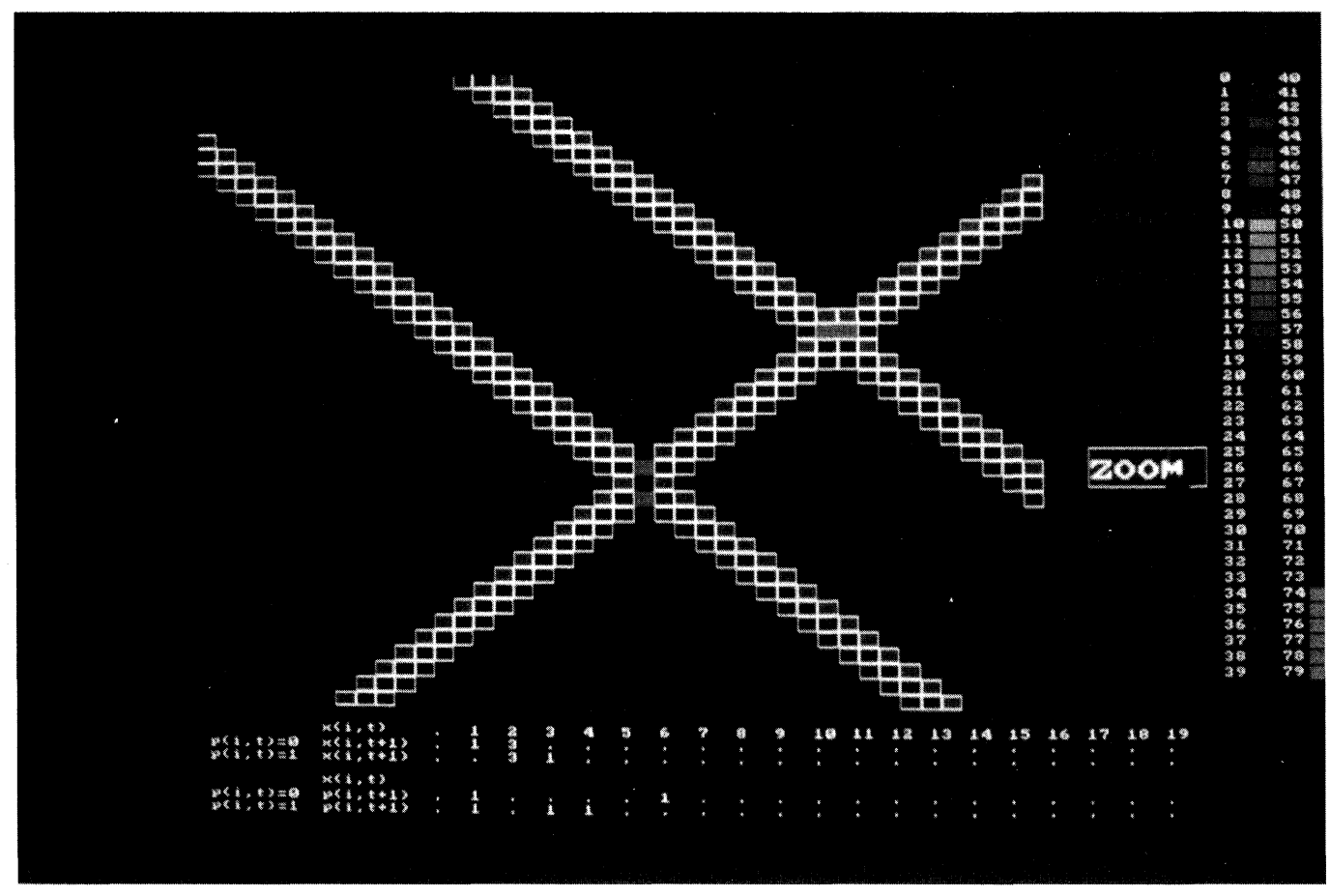

FIGURE 15 Solitary waves crossing each other without any phase shift similar to "classical" waves according to Table X 
TABLE XI

$$
\begin{array}{cllllllll}
p(i, t)=0 & \Sigma(i, t+1) & 0 & 1 & 1 & 2 & 2 & 2 & 3 \\
& \Sigma & 7 & 8 & 9 & 10 & 11 & \ldots \\
p(i, t)=0 & z(i, t+1) & 3 & 3 & 4 & 4 & 4 & \ldots & \\
& \Sigma & 182 & 183 & 184 & 185 & 186 \\
p(i, t)=0 & z(i, t+1) & 61 & 62 & 62 & 62 & 0
\end{array}
$$

In the examples mentioned above, the diffusion coefficient $D$ was either $D=\frac{1}{4}$ or $D=\frac{1}{3}$. But what happens if smaller diffusion coefficients are chosen? Let $D$ be written in the form $D=1 / B$ with $B \geq 3$, so that for any Laplacian diffusion equation

$$
\begin{aligned}
\hat{z}(i, t+1)=z(i, t) & +\frac{1}{B}(z(i-1, t)-2 z(i, t) \\
& +z(i+1, t)) ; \quad\left[\hat{z}_{0}^{1}\right]=z_{0}^{1},
\end{aligned}
$$

one can find a transformation into the averaging procedure. Let us take the abbreviations $z_{0}^{1} \equiv$ $z(i, t+1), z_{0} \equiv z(i, t), z_{-} \equiv z(i-1, t)$ and $z_{+} \equiv$ $z(i+1, t)$. The diffusion equation can now be written in the form

$$
\hat{z}_{0}^{1}=z_{0}+\frac{1}{B}\left(z_{-}-2 z_{0}+z_{+}\right)
$$

and the averaging procedure is described by

$$
\hat{z}_{0}^{1}=\frac{1}{B}\left(z_{-}+(B-2) z_{0}+z_{+}\right) .
$$

But if $B$ becomes smaller than three: $B<3$, and $D>\frac{1}{3}$ respectively, the Laplace diffusion takes the form:

$$
\begin{aligned}
& \hat{z}_{0}^{1}=3 D z_{0}+\frac{1}{B}\left(z_{-}-2 z_{0}+z_{+}\right), \\
& \hat{z}_{0}^{1}=z_{0}+\frac{1}{B}\left(z_{-}-2 z_{0}+z_{+}\right)+(3 D-1) z_{0} .
\end{aligned}
$$

Formally, this means that the classic diffusion is superimposed by a production which is linearly dependent on the state $z_{0} \equiv z(i, t)$ of the cell under consideration. Meinhardt [1] used such linear terms $r_{a} a$ and $r_{b} b$ in his system of differential equations mentioned in the Introduction.

For example, if $D=\frac{1}{2}$ and $B=2$ respectively, this leads to a transformation rule

$$
z_{0}^{1}=[D \Sigma]=\left[\frac{1}{2} \Sigma\right] ; \quad b=0
$$

of the cellular automaton:

TABLE XII

$$
\begin{array}{lcccccccc}
\Sigma & 0 & 1 & 2 & 3 & 4 & 5 & 6 & \\
z(i, t+1) & 0 & 0 & 1 & 1 & 2 & 2 & 3 & \\
& & & & & & & & \\
\Sigma & 7 & 8 & 9 & 10 & 11 & 12 & \ldots \\
z(i, t+1) & 3 & 4 & 4 & 5 & 5 & 6 & \ldots
\end{array}
$$

This produces a solitary wave with a step-like profile. The temporal development of this profile posseses a complex but periodic structure (see Fig. 16).

Starting a more sophisticated cellular vector automaton with such a period two sequence

$$
0,0,1,1,2,2,3,3, \ldots
$$

periodic string of pearls are generated in the interval between the leading front waves (Fig. 17):

\section{TABLE XIII}

$$
\begin{array}{lllllllll} 
& \Sigma & 0 & 1 & 2 & 3 & 4 & 5 & 6 \\
p(i, t)=0 & z(i, t+1) & 0 & 0 & 1 & 1 & 2 & 2 & 3 \\
p(i, t)=1 & z(i, t+1) & 0 & 0 & \ldots & & & & \\
p(i, t)=0 & p(i, t+1) & 0 & 0 & \ldots & & & & \\
p(i, t)=1 & p(i, t+1) & 0 & 0 & \ldots & & & & \\
& \Sigma & \ldots & 47 & 48 & 49 & 50 & \ldots \\
p(i, t)=0 & z(i, t+1) & \ldots & 23 & 24 & 24 & 25 & \ldots \\
p(i, t)=1 & z(i, t+1) & & & & & & \\
p(i, t)=0 & p(i, t+1) & \ldots & 0 & 1 & 1 & 1 & \ldots \\
p(i, t)=1 & p(i, t+1) & & & & & &
\end{array}
$$




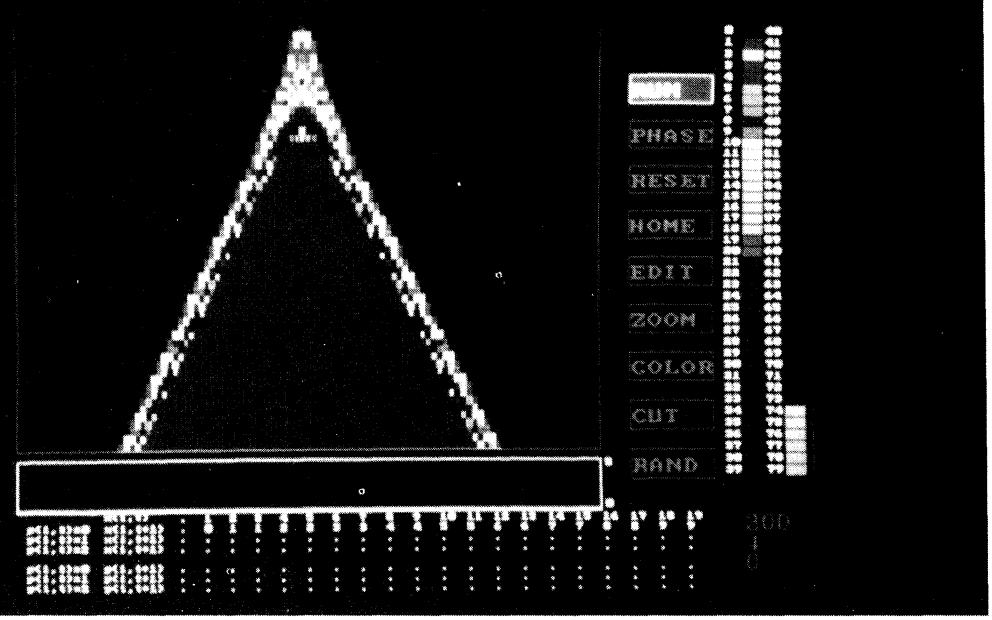

FIGURE 16 Periodic structure of the front according to Table XII. The automaton was started with one cell in the state $\vec{z}(j, 0)=\left(\begin{array}{l}4 \\ 0\end{array}\right)$.

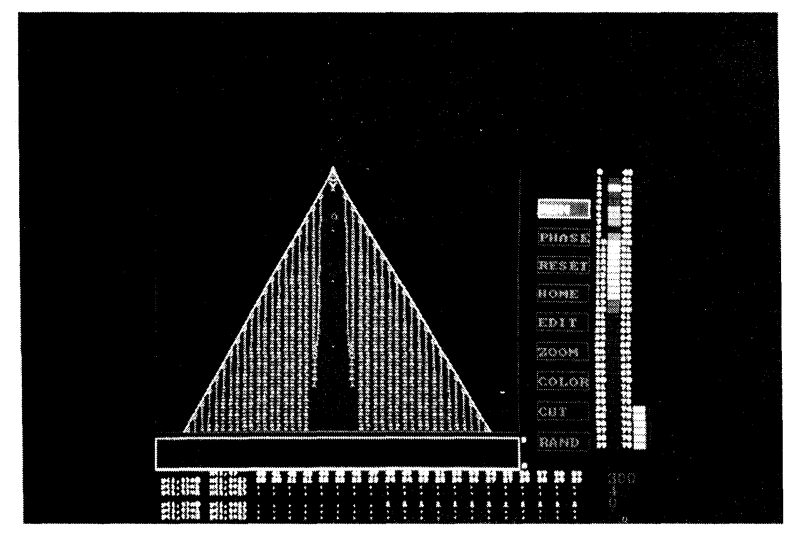

FIGURE 17 Part of the spatio-temporal development of the automaton in Table XIII with a periodic "string of pearls" pattern.

whereas a later switch from $p(i, t)=0$ to $p(i, t+1)=1$ creates chaotic strings of pearls if one starts with one cell $j$ in the state $\vec{z}(j, 0)=\left(\begin{array}{l}8 \\ 0\end{array}\right)$ and $\vec{z}(i \neq j, 0)=\left(\begin{array}{l}0 \\ 0\end{array}\right)$ (Fig. 18):

\section{TABLE XIV}

$\begin{array}{lllllllll} & \Sigma & 0 & 1 & 2 & 3 & 4 & 5 & 6 \\ p(i, t)=0 & z(i, t+1) & 0 & 0 & 1 & 1 & 2 & 2 & 3 \\ p(i, t)=1 & z(i, t+1) & 0 & 0 & \ldots & & & & \\ p(i, t)=0 & p(i, t+1) & 0 & 0 & \ldots & & & & \\ p(i, t)=1 & p(i, t+1) & 0 & 0 & \ldots & & & & \\ & & & & & & & & \\ & \Sigma & \ldots & 54 & 55 & 56 & 57 & \ldots \\ p(i, t)=0 & z(i, t+1) & \ldots & 27 & 27 & 28 & 28 & \ldots \\ p(i, t)=1 & z(i, t+1) & & & & & & \\ p(i, t)=0 & p(i, t+1) & \ldots & 0 & 1 & 1 & 1 & \ldots \\ p(i, t)=1 & p(i, t+1) & & & & & & \end{array}$

Furthermore, changing the "diffusion" by increasing $D$ and hence the state-dependent production $(3 D-1) z_{0}$, for example, one obtains

$$
z_{0}^{1}=[\Sigma-1] \text { and } z_{0}^{1}=0 \quad \text { if } \Sigma=0 .
$$




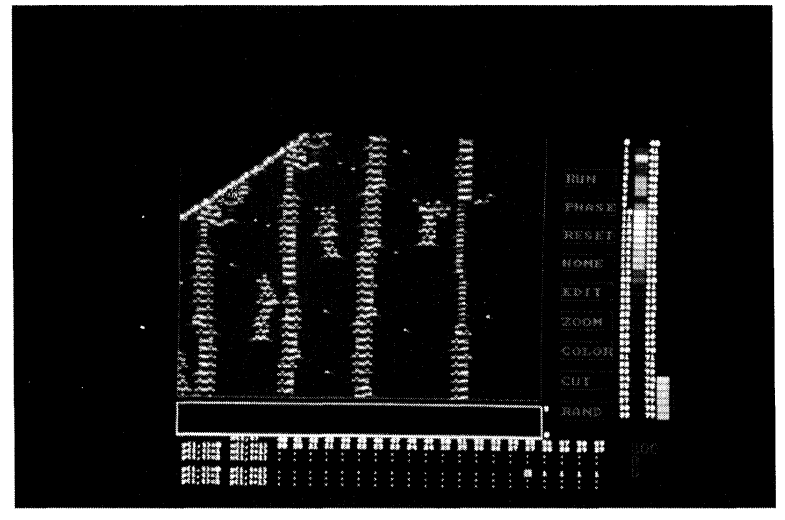

FIGURE 18 Chaotic "string of pearls" generated by the automaton in Table XIV.

\section{TABLE XV}

$$
\begin{array}{lcccccccc}
\Sigma & 0 & 1 & 2 & 3 & 4 & 5 & 6 & \\
z(i, t+1) & 0 & 0 & 1 & 2 & 3 & 4 & 5 & \\
\Sigma & 7 & 8 & 9 & 10 & 11 & 12 & \ldots \\
z(i, t+1) & 6 & 7 & 8 & 9 & 10 & 11 & \ldots
\end{array}
$$

Since $\Sigma=z_{-}+z_{0}+z_{+}$, this transformation rule is equivalent to the reaction diffusion equation

$$
z_{0}^{1}=3 D z_{0}+D\left(z_{-}-2 z_{0}+z_{+}\right)-1
$$

and for $D=1$

$$
z_{0}^{1}=z_{0}+D\left(z_{-}-2 z_{0}+z_{+}\right)+2 z_{0}-1
$$

respectively. The corresponding automaton creates a step-like solitary wave, the temporal development of which seems to be chaotic. By introducing this rule into the vector automaton model

\section{TABLE XVI}

$$
\begin{array}{llllllll} 
& \Sigma & 0 & 1 & 2 & 3 & 4 & 5 \\
p(i, t)=0 & z(i, t+1) & 0 & 0 & 1 & 2 & 3 & 4 \\
p(i, t)=1 & z(i, t+1) & 0 & 0 & \ldots & & & \\
p(i, t)=0 & p(i, t+1) & 0 & 0 & \ldots & & \\
p(i, t)=1 & p(i, t+1) & 0 & 0 & \ldots & &
\end{array}
$$

$$
\begin{array}{llllllll} 
& \Sigma & 6 & \ldots & 49 & 50 & 51 & \ldots \\
p(i, t)=0 & z(i, t+1) & 5 & \ldots & 48 & 49 & 0 & \ldots \\
p(i, t)=1 & z(i, t+1) & & & & & & \\
p(i, t)=0 & p(i, t+1) & & \ldots & 0 & 1 & 1 & \ldots \\
p(i, t)=1 & p(i, t+1) & & & & & &
\end{array}
$$

and starting with one cell $j$ in the state $\vec{z}(j, 0)=\left(\begin{array}{l}8 \\ 1\end{array}\right)$, and $\vec{z}(i \neq j, 0)=\left(\begin{array}{l}0 \\ 0\end{array}\right)$, the pattern in the inner part of the triangle in the two-dimensional space-time becomes turbulent (Fig. 19). We have chosen this term with reference to the work of Mikhailow et al. [8] who observed similar patterns when investigating the complex Ginzburg-Landau equation.

Playing around with the transformation rules one may ask what happens if one uses an inverse sequence like

$$
\vec{z}(i, t+1)= \begin{cases}10-\Sigma & \text { if } 0<\Sigma<10 \\ 0 & \text { if } \Sigma=0 \text { or } \Sigma \geq 10 .\end{cases}
$$

\section{TABLE XVII}

$$
\begin{array}{llllllll} 
& \Sigma & 0 & 1 & 2 & 3 & 4 & 5 \\
p(i, t)=0 & z(i, t+1) & 0 & 9 & 8 & 7 & 6 & 5 \\
p(i, t)=1 & z(i, t+1) & 0 & \ldots & & & & \\
p(i, t)=0 & p(i, t+1) & 0 & \ldots & & & & \\
p(i, t)=1 & p(i, t+1) & 0 & \ldots & & & & \\
& \Sigma & 6 & 7 & 8 & 9 & 10 & \ldots \\
p(i, t)=0 & z(i, t+1) & 4 & 3 & 2 & 1 & 0 & \ldots \\
p(i, t)=1 & z(i, t+1) & 0 & \ldots & & & & \\
p(i, t)=0 & p(i, t+1) & 0 & \ldots & & & & \\
p(i, t)=1 & p(i, t+1) & 0 & \ldots & & & &
\end{array}
$$

This means that for larger states there is a strong diffusion but almost no production, whereas for smaller states there is a strong production but diffusion can be neglected. This transformation rule simulates the competition between an inhibition for higher concentrations and a strong autocatalysis for low concentrations (see Fig. 20).

The patterns, produced by this automaton are very sophisticated even if one starts with only 


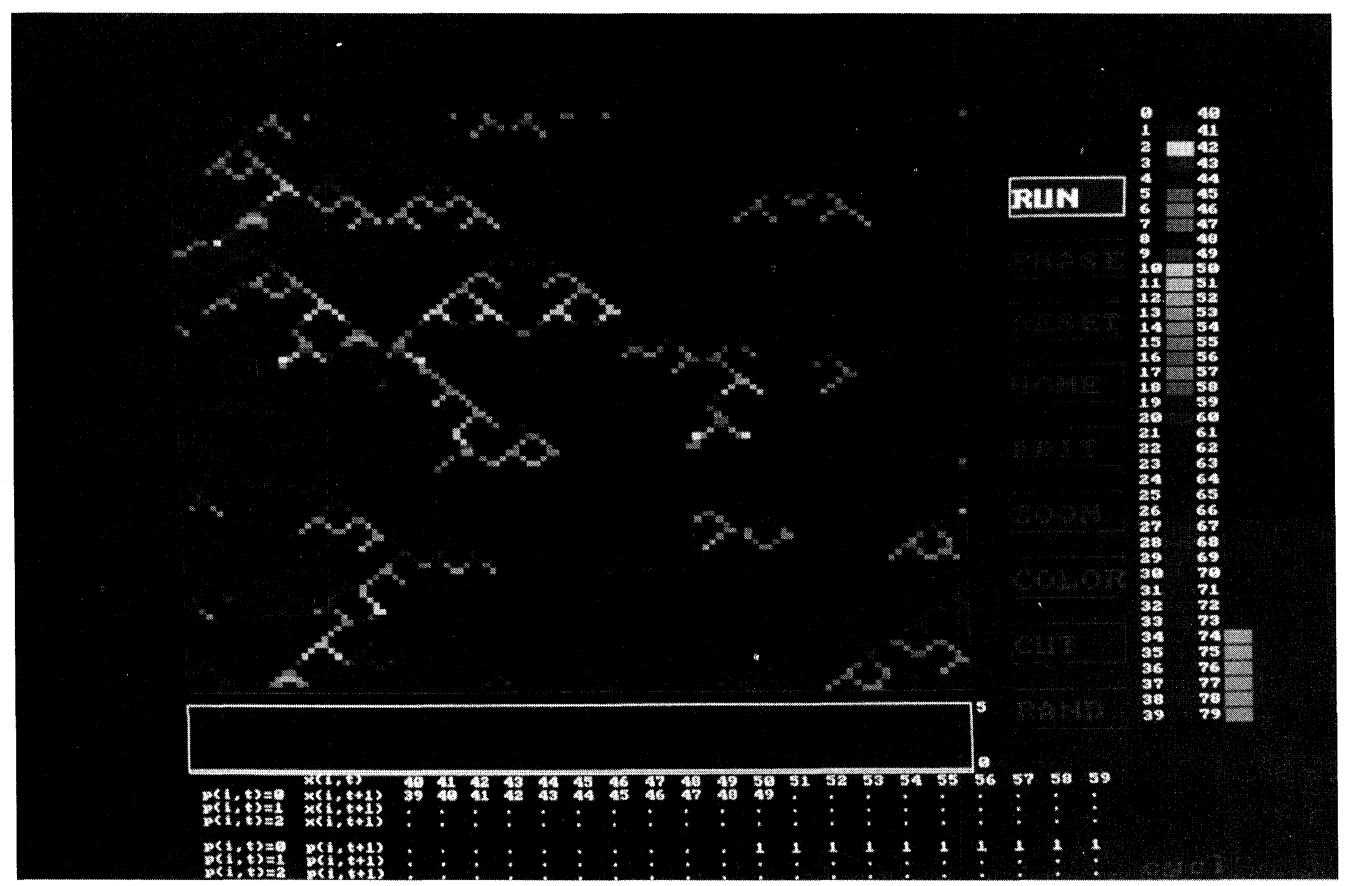

FIGURE 19 "Turbulent" pattern in the temporal development of the one-dimensional automaton according to Table XVI (see $[8])$.

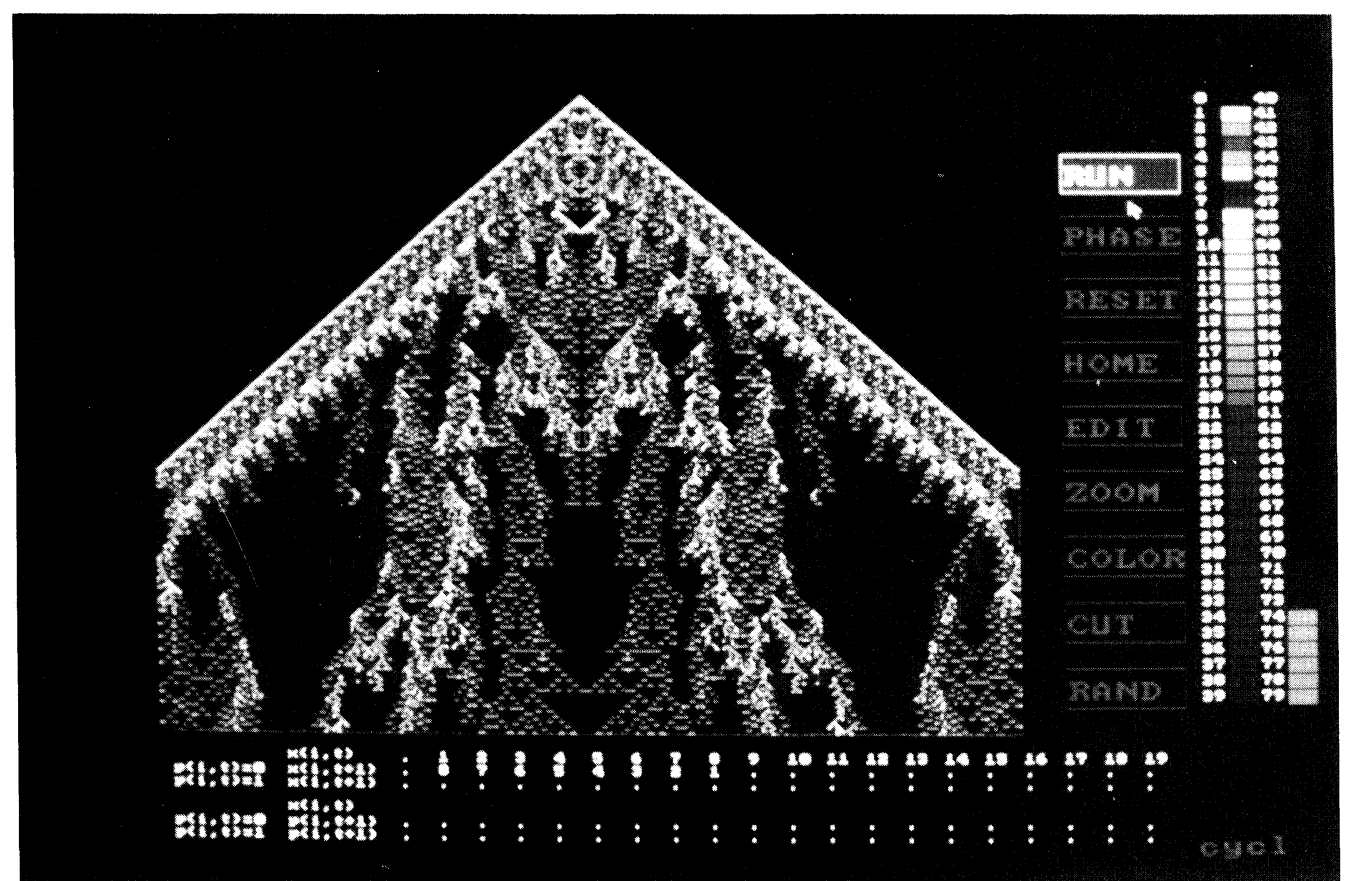

FIGURE 20 Pattern of the "inverse" automaton rule in Table XVII. This pattern resembles the pattern of the spatio-temporal intermittency discussed by Miguel [9]. 


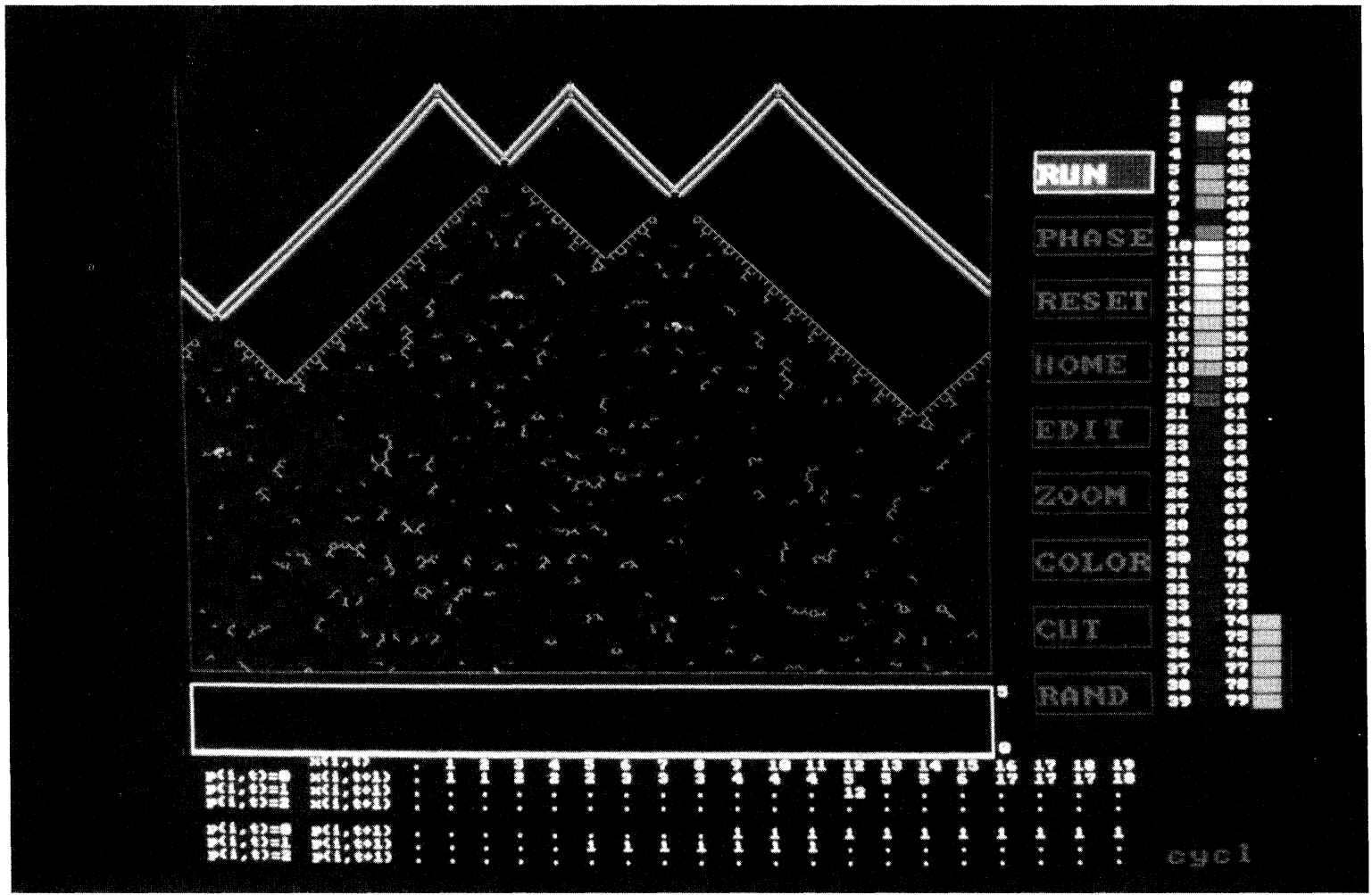

FIGURE 21 "Turbulent" pattern created by the collision of solitary waves according to Table XVIII.

one cell. The patterns resemble very much the patterns which are known for spatio-temporal intermittency [9] in coupled complex GinzburgLaudau equations in the one-dimensional space [10].

We set out to elaborate an automaton producing solitary waves which will create all the fantastic spatio-temporal phenomena if they collide. We now have all the ingredients for its construction. If the concentration states of the cells are small, they should diffuse classically, with the diffusion coefficient $D \leq \frac{1}{3}$. However, if the concentration states of the cells are large enough, an autocatalytic production should govern the behaviour of the system. Through the collision of the low-state solitary waves, states become neigbouring thus throwing the system into the autocatalytic regime. The following transformation rule will create such a behaviour (Fig. 21):
TABLE XVIII

$$
\begin{array}{lllllllll} 
& \Sigma & 0 & 1 & 2 & 3 & 4 & 5 & \\
p(i, t)=0 & z(i, t+1) & 0 & 1 & 1 & 2 & 2 & 2 & \\
p(i, t)=1 & z(i, t+1) & 0 & \ldots & & & & \\
p(i, t)=0 & p(i, t+1) & 0 & \ldots & & & & \\
p(i, t)=1 & p(i, t+1) & 0 & \ldots & & 0 & 1 & \\
& \Sigma & 6 & 7 & 8 & 9 & 10 & 11 & \\
p(i, t)=0 & z(i, t+1) & 3 & 3 & 3 & 4 & 4 & 4 & \\
p(i, t)=1 & z(i, t+1) & & & & & 0 & \\
p(i, t)=0 & p(i, t+1) & & & 0 & 1 & 0 & \ldots & \\
p(i, t)=1 & p(i, t+1) & 1 & 1 & 1 & 1 & 1 & 0 & \\
& \Sigma & 12 & 13 & 14 & 15 & 16 & 17 \\
p(i, t)=0 & z(i, t+1) & 5 & 5 & 5 & 6 & 17 & 17 \\
p(i, t)=1 & z(i, t+1) & 12 & 0 & \ldots & & & \\
p(i, t)=0 & p(i, t+1) & 0 & \ldots & & & & \\
p(i, t)=1 & p(i, t+1) & 0 & \ldots & & & &
\end{array}
$$




$$
\begin{array}{llllllll} 
& \Sigma & 18 & 19 & 20 & 21 & 22 & \ldots \\
p(i, t)=0 & z(i, t+1) & 17 & 18 & 19 & 20 & 21 & \ldots \\
p(i, t)=1 & z(i, t+1) & 0 & \ldots & & & & \\
p(i, t)=0 & p(i, t+1) & 0 & \ldots & & & & \\
p(i, t)=1 & p(i, t+1) & 0 & \ldots & & & &
\end{array}
$$

If the low-state solitary waves collide, they create a turbulent system in the inner part of the spreading "reflected" waves. Moreover, there are transformation rules (Table XIX), constructed in a similar manner, which create turbulent areas spreading with half the velocity of the original solitary waves (Fig. 22):

\section{TABLE XIX}

$$
\begin{array}{cccccccc} 
& \Sigma & 0 & 1 & 2 & 3 & 4 & 5 \\
p(i, t)=0 & z(i, t+1) & 0 & 1 & 1 & 2 & 2 & 2 \\
p(i, t)=1 & z(i, t+1) & 0 & \ldots & & & & \\
p(i, t)=0 & p(i, t+1) & 0 & \ldots & & & & \\
p(i, t)=1 & p(i, t+1) & 1 & 1 & 1 & 1 & 1 & 1
\end{array}
$$

$$
\begin{array}{rlccccc} 
& \Sigma & 6 & 7 & 8 & 9 & 10 \\
p(i, t)=0 & z(i, t+1) & 3 & 3 & 3 & 4 & 4 \\
p(i, t)=1 & z(i, t+1) & & & & 0 \\
p(i, t)=0 & p(i, t+1) & & 0 & 1 & 1 & 1 \\
p(i, t)=1 & p(i, t+1) & 1 & 1 & 1 & 1 & 1 \\
& \Sigma & 11 & 12 & 13 & 14 \\
p(i, t)=0 & z(i, t+1) & 5 & 5 & 6 & 6 \\
p(i, t)=1 & z(i, t+1) & 0 & 8 & 0 & \ldots \\
p(i, t)=0 & p(i, t+1) & 1 & 1 & 1 & 1 \\
p(i, t)=1 & p(i, t+1) & 1 & 0 & \ldots & \\
& \Sigma & \ldots & 45 & 46 & \\
p(i, t)=0 & z(i, t+1) & \ldots & 22 & 22 & \\
p(i, t)=1 & z(i, t+1) & & & & \\
p(i, t)=0 & p(i, t+1) & \ldots & 1 & 1 & \\
p(i, t)=1 & p(i, t+1) & & & &
\end{array}
$$

Even the "strings of pearls" (Fig. 23) can be generated in a similar way by the automaton (Table XX):

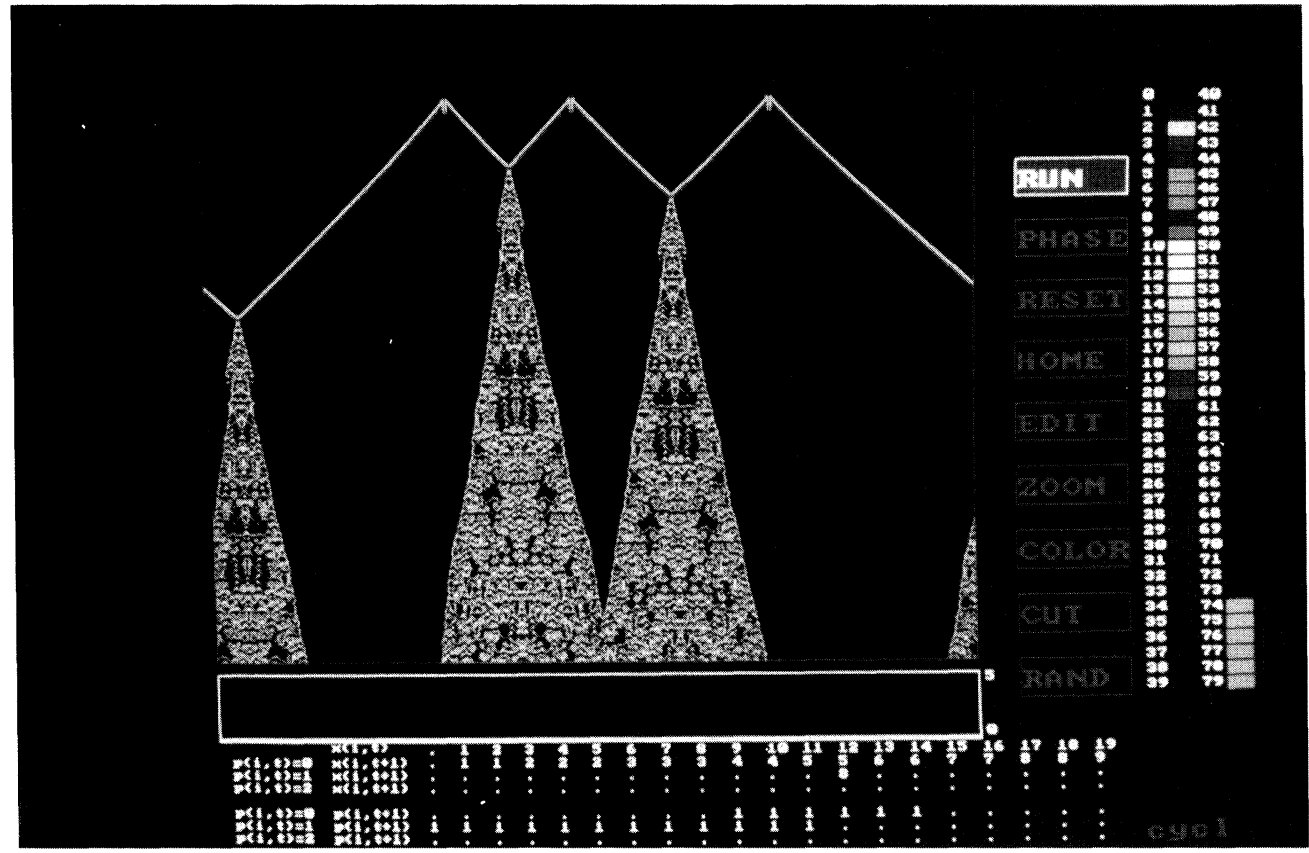

FIGURE 22 Spreading of the "turbulent" interval with half the velocity of the original colliding solitary waves according to Table XIX. 
TABLE XX

$$
\begin{array}{llllllll} 
& \Sigma & 0 & 1 & 2 & 3 & 4 & 5 \\
p(i, t)=0 & z(i, t+1) & 0 & 1 & 1 & 2 & 2 & 2 \\
p(i, t)=1 & z(i, t+1) & 0 & \ldots & & & & \\
p(i, t)=0 & p(i, t+1) & & & & & & \\
p(i, t)=1 & p(i, t+1) & & & & & \\
& \Sigma & 6 & 7 & 8 & 9 & 10 \\
p(i, t)=0 & z(i, t+1) & 3 & 3 & 3 & 4 & 5 \\
p(i, t)=1 & z(i, t+1) & 0 & \ldots & & & \\
p(i, t)=0 & p(i, t+1) & & & & & \\
p(i, t)=1 & p(i, t+1) & & & & & \\
& \Sigma & 11 & 12 & 13 & \ldots & \\
p(i, t)=0 & z(i, t+1) & 5 & 6 & 6 & \ldots & \\
p(i, t)=1 & z(i, t+1) & 0 & 20 & 0 & \ldots & \\
p(i, t)=0 & p(i, t+1) & & & &
\end{array}
$$

\section{F CONCLUSION}

We have shown only a very few examples in order to demonstrate the power of our vector automaton model for simulating a high variety of collision patterns of solitary waves. Our aim was neither to present a complete classification of the possible patterns which can be achieved by this type of automata, nor to simulate all natural sea shell patterns.

We pursued the idea that the natural patterns among biological populations of neighbouring individual cells can be understood by means of discrete mathematical tools such as cellular vector automata. The classical ideas of diffusion and production can easily be induced using these automata models.

As we have shown, a new idea was generated in the process. The dynamics of localized excitations created by the collision of solitary waves can be described in terms similar to those of

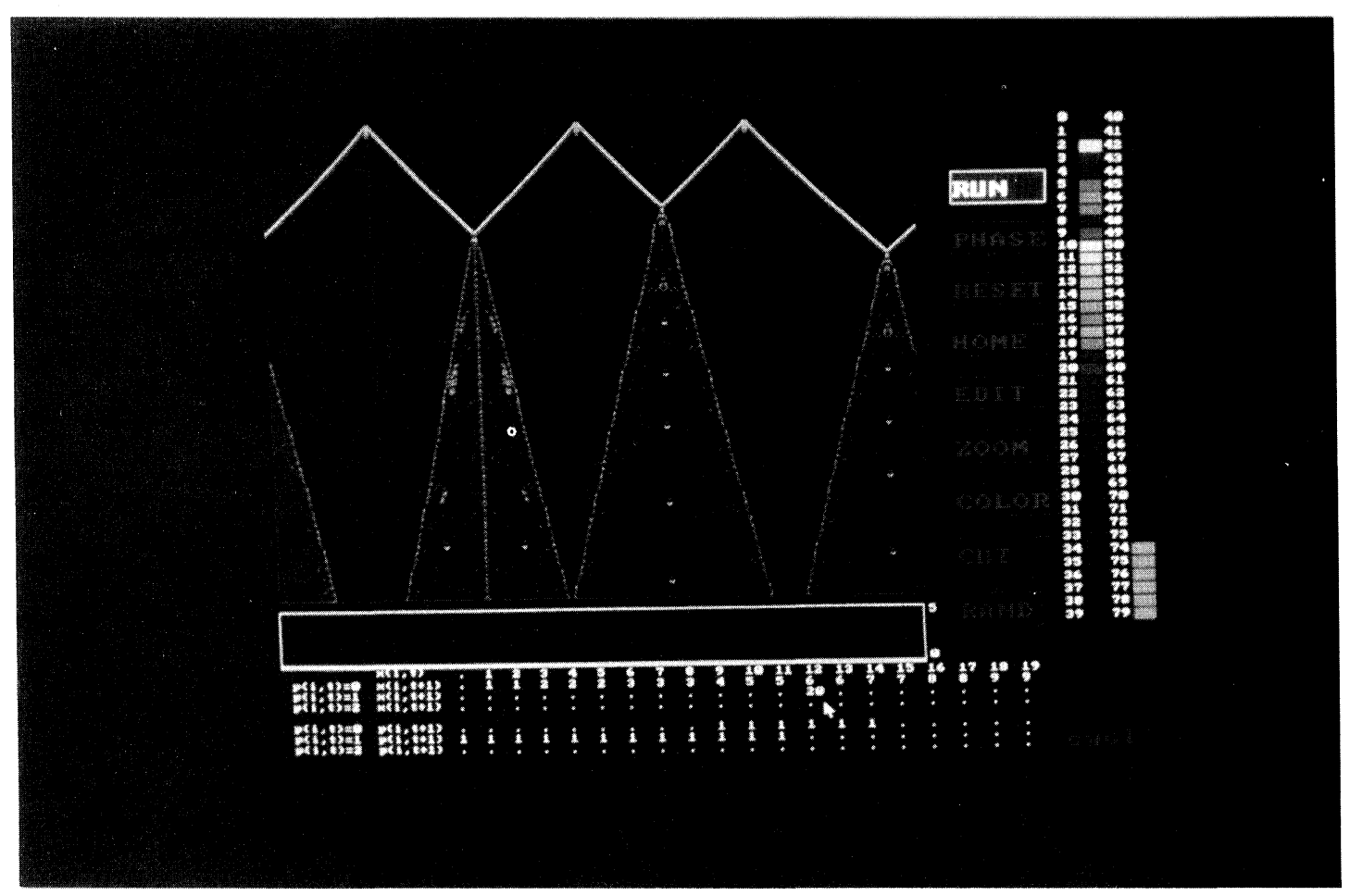

FIGURE 23 "String of pearls" generated by the collision of solitary waves according to Table XX. 


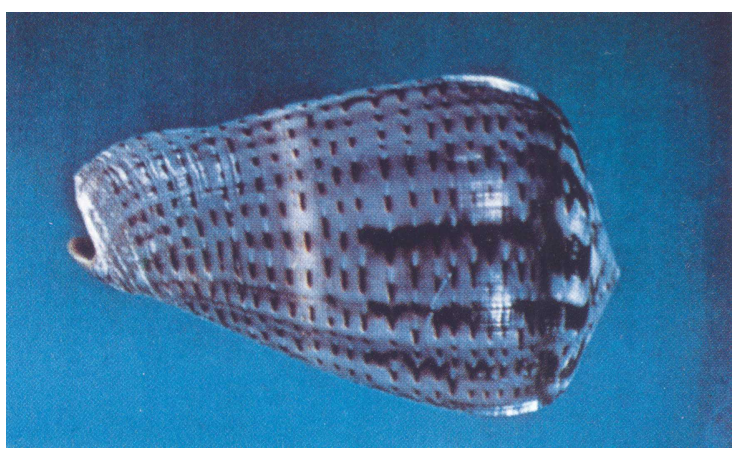

FIGURE 24 A localized oscillating pattern of the cone-shell Glaucus, Solomon Is (with the kind permission of Jerry G. Walls [7d]). See Color Plate V.

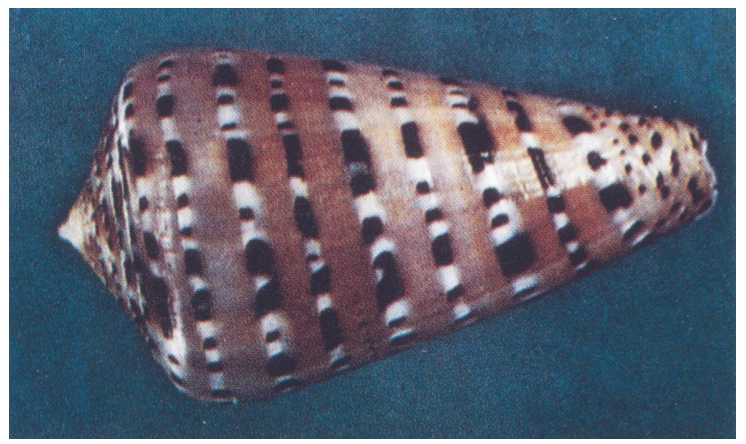

FIGURE 25 A "pearl string" pattern on the cone shell Genuanes, Dhakar, Senegal (with the kind permission of Jerry G. Walls [7e]). See Color Plate VI.

ordinary dynamic systems. There are localized stable stationary states which give rise to the formation of stripes in the $2 D$ - space-time parallel to the direction of growth, as can be observed on the sea shells (Figs. 10, 11).

There also exist localized oscillating patterns (Fig. 24) and localized chaotic patterns (Fig. 25) similar to the pearl string patterns.

A new class of patterns has been found: delocalized states. This means an excitation spreads over the automaton leaving all cells in an excited state. Examples include not only the well known front wave, but many other phenomena as well, such as the spreading of turbulent or spatially intermittent patterns.

Furthermore, there are colliding systems which are spatially and temporally instable. Such sys- tems are classically described by solitons moving in excitable systems.

Between these systems and the spatially and temporally stable systems there exist pulsating systems. If these systems are always pushing out new pairs of solitary waves, they are commonly known as reverberators. But again, this class of automata embraces more behaviour patterns than only the classical ones.

This article provides an initial insight into this fascinating area. Much more work has to be done in the future. We have not investigated the reflection behaviour of the different solitary waves, which will bring us to a much better understanding of natural sea-shell patterns. But this is certainly not the only question we have left aside for the future.

\section{Acknowledgement}

We thank Michael Drosdowski for his support in writing a suitable algorithm.

\section{References}

[1] H. Meinhardt (1995), The Algorithmic Beauty of Sea Shells, Springer-Verlag, Berlin, Heidelberg.

[2] P.J. Plath and J. Schwietering (1992), in: Fractal Geometry and Computer Graphics (Eds. J.L. Encarnacao, H.-O. Peitgen, G. Sakas and G. Englert), Springer-Verlag, Berlin, Heidelberg, pp. 162-172.

[3] M. Gerhardt, H. Schuster and J.J. Tyson (1990), Science 247, 1563-1566.

[4] M. Gerhardt, H. Schuster and J.J. Tyson (1990), Physica D 46, 392-415.

[5] J. Schwietering and P.J. Plath (1992), Wiss. und Fortschritt 42, 73-75.

[6] E. Atlee Jackson (1991), Perspectives of Nonlinear Dynamics, Vol. 1, Cambridge University Press, Cambridge, New York, Melbourne, Sydney, pp. 143.

[7] J.G. Walls, Cone Shells - A Synopsis of the Living Conidae, TFH. Publication Inc., Neptune City, New Jersey, USA; (a) p. 553, (b) p. 357 (c) p. 36 (d) p. 333, and (e) p. 324.

[8] D. Bottogtokh and A. Mikhailow (1995), lecture at the workshop "Complex Pattern in Distributed Active media", Erfurt, Germany, forthcoming.

[9] H. Chaté (1994), Nonlinearity, 7, 185.

[10] M. San Miguel (1995), lecture at the workshop "Complex Pattern in Distributed Active media", Erfurt, Germany, forthcoming.

[11] L.A. Dimitrieva and M.A. Khlabystova (1996), St. Petersburg, Russia, IPRT Reprint No. 104-95 (1995) and No. 109-96. 


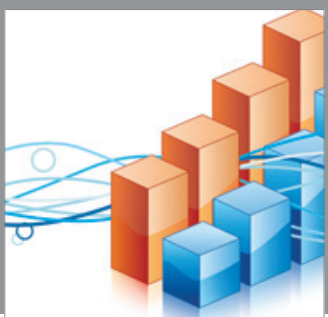

Advances in

Operations Research

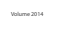

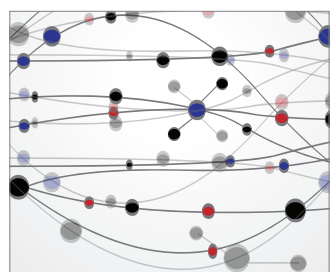

\section{The Scientific} World Journal
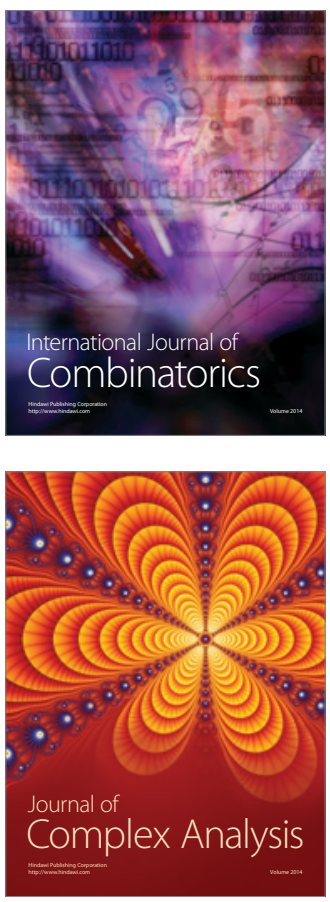

International Journal of

Mathematics and

Mathematical

Sciences
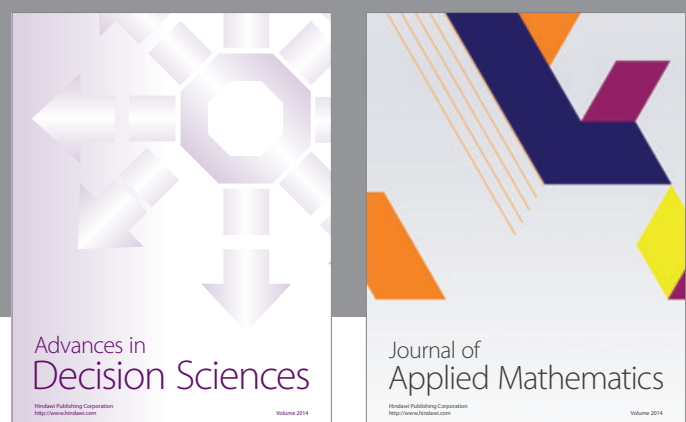

Journal of

Applied Mathematics
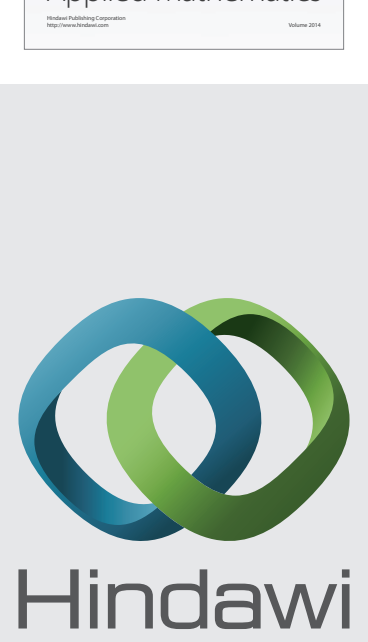

Submit your manuscripts at http://www.hindawi.com
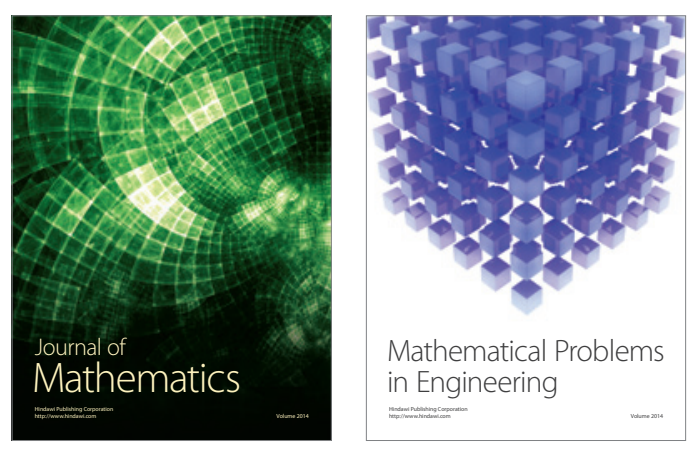

Mathematical Problems in Engineering
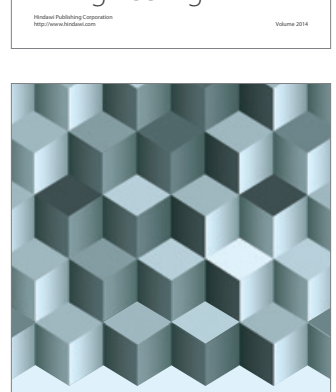

Journal of

Function Spaces
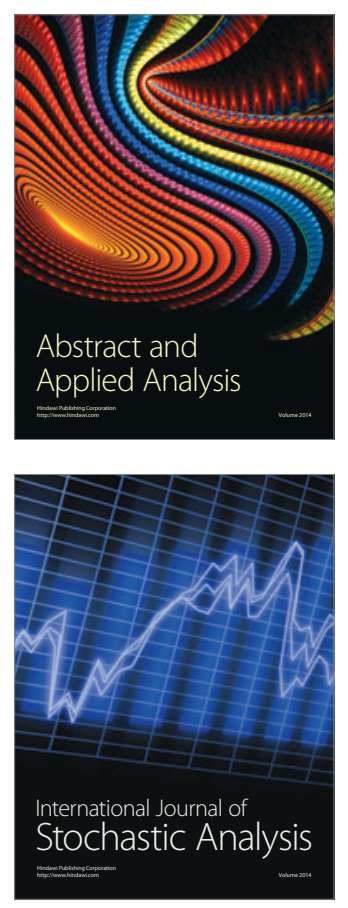

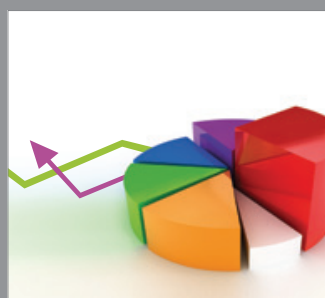

ournal of

Probability and Statistics

Promensencen
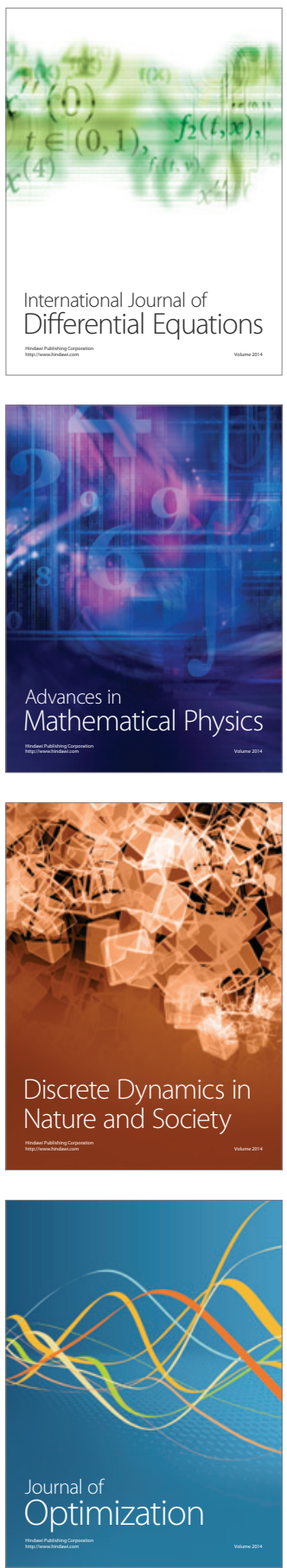